\title{
Interleukin-17 programs liver progenitor cell transformation into cancer stem cells through miR-122 downregulation with increased risk of primary liver cancer initiation
}

\author{
Imène Gasmi ${ }^{1,2}$, Camilia Machou ${ }^{1,2}$, Aurélie Rodrigues ${ }^{1,2}$, Arthur Brouillet ${ }^{1,2}$, Trung Cong Nguyen ${ }^{1,2}$, Benoit \\ Rousseau ${ }^{1,2}$, Adrien Guillot ${ }^{1,2}$, Christophe Rodriguez ${ }^{1,2}$, Vanessa Demontant ${ }^{1,2}$, Yeni Ait-Ahmed ${ }^{1,2}$, Julien \\ Calderaro ${ }^{1,2,4}$, Alain Luciani1,2, Jean-Michel Pawlotsky ${ }^{1,2,5}$, Fouad Lafdil1,2,3凶 \\ 1. Université Paris-Est, UMR-S955, UPEC, F-94000, Créteil, France. \\ 2. INSERM, U955, F-94000, Créteil, France. \\ 3. Institut Universitaire de France (IUF), Paris, F-75231 Cedex 05 France. \\ 4. Département de Pathologies, Hôpital Henri Mondor, Université Paris-Est, Créteil, France. \\ 5. Département de Virologie, Hôpital Henri Mondor, Université Paris-Est, Créteil, France. \\ $\square$ Corresponding author: Prof. Fouad LAFDIL, INSERM U955, Institut Mondor de Recherche Biomédicale, Hôpital Henri Mondor, 94000 Créteil, France. Tel: \\ +33 (0)1 498135 38; Fax: + 33 (0)1 498148 44; E-mail: fouad.lafdil@inserm.fr; fouad.lafdil@u-pec.fr.
}

(c) The author(s). This is an open access article distributed under the terms of the Creative Commons Attribution License (https://creativecommons.org/licenses/by/4.0/). See http://ivyspring.com/terms for full terms and conditions.

Received: 2021.12.23; Accepted: 2021.12.29; Published: 2022.02.21

\begin{abstract}
Chronic inflammation is a key component in the development of virtually all types of primary liver cancers. However, how chronic inflammation potentiates or even may initiate liver parenchymal cell transformation remains unclear. Cancer stem cells (CSCs) represent an exciting target for novel anticancer therapeutic strategies in several types of cancers and were also described in primary liver cancers as tumor initiating cells. Recently, we reported a key role of Interleukin (IL)-17 in Liver Progenitor Cell (LPC) accumulation in preneoplastic cirrhotic livers. In this study, we evidenced in vitro, that long-term stimulation of LPCs with IL-17 led to their transformation into CSCs. Indeed, they acquired CSC-marker expression, and self-renewal properties, showed by their increased capacity to form spheroids. The miRNome analysis revealed that long-term IL-17 treatment of LPCs led to a $90 \%$ decrease in miR-122 expression. In a model using immunodeficient mice, ectopic engraftment of LPCs in an IL-17-enriched environment led to tumor occurrence with an aggressive phenotype. Contrastingly, in a murine model of hepatocellular carcinoma induced by a unique injection of diethyl-nitrosamine associated with chronic administration of carbon tetrachloride, IL-17-deficiency or anti-IL-17 therapy protected mice from liver tumor growth. In conclusion, we showed that a chronic exposure of LPCs to IL-17 cytokine promotes their transformation into CSCs. In addition, we demonstrated that IL-17-neutralizing strategies limit CSC occurrence and liver tumor progression through miR-122 restored-expression.
\end{abstract}

Key words: Liver progenitor cells; Interleukin-17; cancer stem cells; liver cancer, miR-122

\section{Introduction}

Primary liver cancer is the fifth-most frequent cancer worldwide and the second leading cause of cancer-related death [1]. Hepatocellular carcinoma (HCC) accounts for $80 \%$ of all primary liver cancers and represents the leading cause of death in patients with chronic liver diseases. Intrahepatic cholangiocarcinoma (ICC) arising from bile ducts within the liver parenchyma is the second most common $(15 \%)$ primary hepatic malignancy after HCC [2]. Despite significant advances in liver cancer diagnosis and therapy over the past decade, the current anticancer treatments remain poorly effective in advanced stages of the disease [3]. 
HCC and ICC have been considered as distinct tumors that originate from different hepatic cells (i.e. hepatocytes vs biliary cells, respectively). However, emerging evidence suggest that both primary liver cancers could also originate from a common tumor initiating cell population [4,5]. Interestingly, Cancer Stem Cells (CSCs) have recently sparked the interest of scientists because of their capacity to initiate and enhance tumor progression of several cancers [6]. CSCs have been found in virtually all types of cancers including HCC or ICC and their presence is generally associated with poor outcomes [7], which contribute to the failure of conventional targeted cancer therapies [8]. However, the origin of CSCs, and the underlying mechanisms by which they develop in the liver remain unclear.

Liver Progenitor Cells (LPCs) are defined as intrahepatic quiescent cells that are activated in chronic liver diseases and participate to liver regeneration when the proliferation of remaining hepatocytes is impaired [9]. They are bipotent cells that could differentiate into either hepatocytes or biliary cells. LPC accumulation, referred to as ductular reaction, is frequently observed particularly in cirrhotic livers with a worse prognosis [10]. Interestingly, it has been observed that 28 to $50 \%$ of HCC express progenitor/stem cell markers [11]. Besides, ICC is thought to originate from biliary cells in tight interaction with canals of Hering where the LPC compartment is located [12]. For those reasons, it is accepted that LPCs have the potential to become tumor initiating cells because of their likelihood to transform into CSCs that ultimately evolve into heterogeneous lineages of cancer cells [5, 9, 13]. Oncogenic processes leading to the transformation of LPCs into CSCs remain poorly understood.

Primary liver cancers occur in $90 \%$ of cases in an inflammatory context present in virtually all chronic liver diseases [14]. The inflammatory response plays a key role in the activation of the LPC compartment [10], while chronic and persistent inflammatory response may favor LPC transformation into CSCs [5]. In this inflammatory context, Th17 cells are crucial components among infiltrating immune cells in HCC [15] and numerous studies revealed that Interleukin17A (IL-17), a cytokine mainly produced by Th17 cells, can directly act on stem cells to promote their proliferation, and thus, tissue repair. However, IL-17 may also participate in cancer initiation, tumor progression and metastasis [16]. We recently reported a positive correlation between the number of IL-17-infiltrating cells and the number of LPCs accumulating in human cirrhotic livers from various etiologies [17]. In addition, a high IL-17 expression has been associated with a poor prognosis of HCC
[18], along with an increased hepatic tumor growth and metastasis [19]. Recently, plasma IL-17 concentration has been identified as a predictor of subsequent HCC occurrence in liver cirrhosis patients [20], but whether and how IL-17 could play a role in the initiation of liver tumorigenesis remains unclear.

In this study, we hypothesized that IL-17 could participate in liver cancer initiation by promoting LPC transformation into a CSC phenotype. We showed in vitro that long-term exposure of LPCs to IL-17 leads to their transformation into CSCs, and we evidenced in two murine models that IL-17 neutralization limits cancer initiation and progression. These results suggest that IL-17-neutralizing strategies may be used to prevent primary liver cancer development.

\section{Materials and methods}

\section{Human samples}

Forty-five liver tissue samples from previously described patients [17] with diverse chronic liver diseases were analyzed. The study conformed to the ethical guidelines of the 1975 Declaration of Helsinki was approved by the local ethics committee Ile de France I (Institutional Review Board 2017-A01215-48) as required by the French legislation. Blocks of formalin-fixed paraffin-embedded samples from explanted livers were obtained from the Department of Pathology of Henri Mondor University Hospital (Creteil, France). For the assessment of CK19- , IL-17-and CD133-positive cell density, slides were reviewed and scored by two independent evaluators, including a pathologist specialized in liver diseases, using a semi-quantitative score and patients were dichotomized into high versus low density of stained cells for each labeling as previously described [17].

\section{LPC cell lines and culture conditions}

Bipotential Murine Oval Liver cells (BMOL) (kindly provided by Pr George C. Yeoh, School of Medicine and Pharmacology, University of Western Australia) and human HepaRG (obtained from Biopredic International, France) were cultured in a humidified atmosphere at $37^{\circ} \mathrm{C}$ and $5 \% \mathrm{CO}_{2}$ (with or without IL-17) for 10, 20, 30 and 40 days. Recombinant mouse and human IL-17 were purchased from R\&D Systems. For BMOL culture, $50 \mathrm{ng} / \mathrm{ml}$ of recombinant mouse IL-17 was added every three days to William's medium supplemented with 5\% Fetal Calf Serum (FCS), $10 \mu \mathrm{g} / \mathrm{ml}$ insulin and $10 \mathrm{ng} / \mathrm{ml}$ of recombinant mouse Epidermal Growth Factor (EGF). Fifty ng/ml of recombinant human IL-17 was added every 3 days to HepaRG cultured in William's medium supplemented with 10\% FCS, insulin and hydrocortisone hemisuccinate at final concentration of $4 \mu \mathrm{g} / \mathrm{ml}$ and $50 \mu \mathrm{M}$ respectively. 


\section{Animals}

\section{Ectopic allograft of IL-17-expressing LPCs}

For in vivo characterization of IL-17 tumorigenic effect, BMOL cells were genetically modified to constitutively express IL-17 and luciferase genes. A pUNO1-mIL17A plasmid (InvivoGen) was used in co-transfection experiments with the pGL4.51 (Luc2) vector (Promega) using the Lipofectamine ${ }^{\circledR}$ LTX Reagent with PLUSTM Reagent (Life Technologies), and stably expressing LPCs were isolated after 3 weeks of selection by G418 and blasticidin antibiotics. As an empty control vector, pUNO1-mcs plasmid was used. Subcutaneous implantation of $10^{6}$ transfected cells was performed into NOD/SCID mice in accordance with the local Committee of for the Use of Live Animals. In vivo cell expansion was monitored weekly for 12 weeks by bioluminescence. Briefly, mice were intraperitoneally injected with $200 \mu \mathrm{l}$ of 15 $\mathrm{mg} / \mathrm{ml}$ D-luciferin solution. Tumor size and cell density were assessed 10 minutes later using an IVIS spectrum imaging device (Perkin Elmer) under gas anesthesia (2.5\% isoflurane). An auto-drawing feature was used to draw the region of interest (ROI) and the total number of photons emitted per second was calculated.

\section{$\mathrm{DEN}+\mathrm{CCl}_{4}$ murine model}

C57BL/6J (Charles River) and C57BL/6J Il17a ${ }^{\text {tm1Yiw }} /$ Il17a ${ }^{\text {tm1Yiw }}$ (kindly provided by Pr Yoichiro Iwakura) were injected intraperitoneally (i.p.) at 2 weeks of age with diethylnitrosamine (DEN, 25 $\mathrm{mg} / \mathrm{kg}$, Sigma Aldrich). From week 8 of age, only male C57BL/6J mice were injected twice per week with $10 \%$ carbon tetrachloride $\left(\mathrm{CCl}_{4}\right),(2 \mathrm{mg} / \mathrm{kg}$, Sigma Aldrich) for 14 weeks, and the last 6 weeks, mice were treated with $100 \mu \mathrm{g}$ of anti-IL-17 antibodies or its isotype control (BE0173, BE0083, BioXcell) twice per week. Tumor areas were quantified using QuPath, a free open-source software for digital pathology analysis [21].

\section{Sphere forming assay}

BMOL cells were cultured for 30 days in William's medium with $5 \%$ FCS (with or without IL-17 at $50 \mathrm{ng} / \mathrm{mL}$ ) in adherence conditions. Then cells were collected and resuspended in serum-free DMEM/F12 media supplemented with $20 \mathrm{ng} / \mathrm{ml}$ of B27 supplement, recombinant mouse EGF and basic FGF. The cells were subsequently cultured in ultra-low attachment 12-well plates at a density of 5 cells $/ \mu \mathrm{L}$. To test BMOL self-renewal capacity, the spheres were counted and dissociated 7 days later with Accutase enzyme, then seeded again in ultra-low attachment wells at the same density of 5 cells $/ \mu \mathrm{L}$.

\section{Flow cytometry analysis}

For CD133 (Prominin-1) labeling, 1 million cells were resuspended in PBS supplemented with $2 \mathrm{mM}$ EDTA and $0.5 \%$ of bovine serum albumin. Antiprominin-1 coupled with APC or IgG1 control antibodies (Miltenyi) were added in cell suspension and incubated for $15 \mathrm{~min}$ at $4{ }^{\circ} \mathrm{C}$ in the dark. Then the cells were washed and resuspended in PBS supplemented with EDTA and BSA for flow cytometry analysis.

\section{Immunostaining}

Mouse tumors were fixed in $4 \%$ paraformaldehyde and embedded in paraffin. Sections were labeled with primary antibodies against CK-19 (TROMA III, DSHB), AFP (Santa Cruz, sc-8108), and CD133 (Abcam, ab19898) overnight at 4 ${ }^{\circ} \mathrm{C}$. Then, sections were incubated for $45 \mathrm{~min}$ with goat anti-rat IgG Alkaline Phosphatase (MP-5404), donkey anti-goat IgG-B (SC-2042), or horse Anti-Rabbit IgG Peroxidase (Vector, MP-7401), respectively. Detection was performed using a liquid permanent red (Dako, K0640) for CK-19 staining. For AFP and CD133 immunostaining, a Vector ImmPRESS Reagent, Peroxidase with DAB exposure was used. HE and Red Sirius staining were performed in the Henri Mondor pathology department. Coloration staining and immunostaining quantification were performed using ImageJ software (NIH) by means of color deconvolution plugin followed by MRI Fibrosis Tool [22] for Sirius red coloration, and IHC profiler plugin for AFP and CD133 staining.

\section{RNA analysis}

Total RNA was isolated from lysed cells using RNeasy Mini kit (Qiagen) according to the manufacturer's protocol. The RNA concentration in each sample was assessed using a Nanodrop ND-1000 spectrophotometer (Thermoscientific). Two $\mu \mathrm{g}$ of RNA was reverse transcribed from random primers using a High capacity kit (AB applied). Cd133, Epcam, Aldh, Klf4, Gpc3, Afp, Cyclin D1, Cyclin E, P21, Zeb1, Snail, Ck19, Col1, Thy1, Cd44, Lgr5 and Asma transcript levels were measured by quantitative reverse transcriptase polymerase chain reaction (RT-qPCR) using a LightCycler 480 (Roche). DNA amplification was performed using $25 \mathrm{ng}$ of cDNA, $10 \mu \mathrm{M}$ forward and reverse primers and the Quantitect SYBR green PCR mix (Qiagen).

\section{MicroRNA extraction and analysis}

Extraction of total RNA and microRNA was performed using the QIAsymphony RNA Kit (Qiagen) according to the manufacturer's protocol. In 
addition, the samples were treated with DNAse to avoid contamination by genomic or mitochondrial DNA. A total of $10 \mathrm{ng}$ of RNA was used for cDNA preparation using the MiScript II RT Kit (Qiagen) and the mmu-miR-122 transcript levels were measured by qPCR on the LightCycler480 (Roche), using the miScript SYBR Green PCR kit with mmu-miR-122 miScript primer assay (Qiagen). According to the supplier's protocol, MicroRNA expression was determined as absolute quantification. To estimate the absolute copy number of miR-122, a standard range was established by 10-fold dilution over 10 points using mus musculus miR-122 (mmu-miR-122) mimic (Dharmacon). The dilution range used for reversetranscription with miScript Reverse Transcription kit (Qiagen) started from $7.1310^{11}$ copies per ng of total RNA for mmu-miR-122 mimic. Copy number of miR-122 per ng of total RNA in each sample was determined by plotting $\mathrm{Ct}$ values in the mmu-miR-122 standard curves.

\section{Cell proliferation assay}

Cell proliferation assay was performed on BMOL cells pretreated with $50 \mathrm{ng} / \mathrm{mL}$ IL-17 for 20 and 40 days using 3-(4,5-dimethylthiazol-2-yl)-5(3-carboxymethoxyphenyl)-2-(4-sulfophenyl)-2Htetrazolium (MTS) purchased from Promega. Two thousand five hundred cells per well were seeded in $200 \mu \mathrm{L}$ William's medium supplemented with 5\% FCS, in 96-well plates in six replicates and incubated for 6 hours, 1, 3, 7 and 8 days. The medium was changed every 2 days without adding recombinant mouse IL-17. After the treatment period, the medium was replaced with $100 \mu \mathrm{L}$ DMEM without phenol red (Gibco) and $20 \mu \mathrm{L}$ MTS agent was added to each well. The assay is based on the bio-reduction of MTS by live cells into a colored formazan product that can be quantified by absorbance measurement at $490 \mathrm{~nm}$ (Biotek ELX808LBS reader), and optical density (OD) values normalized with control BMOL.

\section{Protein analysis}

Total proteins from BMOL cells cultured for 20 or 40 days in William's medium with 5\% FCS with or without IL-17 were extracted with ice-cold RIPA lysis buffer and quantified with the BCA kit (Pierce). Proteins were electrophoresed on SDS-PAGE gels and transferred to a nitrocellulose membrane (Invitrogen) after blocking with $5 \%$ BSA. Ten $\mu \mathrm{g}$ of proteins were added to each well. Protein detection was done using the following antibodies: anti-cyclin D, anti-cyclin E, anti-p21 from Santa Cruz Biotechnologies, antiRetinoblastoma (RB), anti-phosphorylated RB and anti-c-Raf from Cell Signaling Technology and anti- $\beta$-actin from Sigma-Aldrich. The membrane was incubated with the secondary antibodies (Horseradish peroxidase) and signals were detected with ECL reagent (GE Healthcare). The band intensity was measured by using Image J software (NIH).

\section{In situ Proximity Ligation Assay}

BMOL cells $(20,000$ cells) were treated with 50 $\mathrm{ng} / \mathrm{mL}$ of IL-17 for 40 days and seeded in Lab-Tek chambers overnight at $4{ }^{\circ} \mathrm{C}$. Adherent cells were fixed with $4 \%$ PFA in PBS and saturated with $0.1 \%$ BSA fraction $\mathrm{V}$ at $37^{\circ} \mathrm{C}$ for an hour. Cells were incubated with anti-mouse cyclin E, and anti-mouse p21 or with anti-mouse cyclin D and anti-mouse p21 monoclonal antibodies, each at a 1:50 dilution for an hour at $37^{\circ} \mathrm{C}$. Ligation, amplification and detection were performed with Duolink in situ Orange Starter Kit. Anti-Mouse/ Rabbit were used according to the manufacturer's instructions and images were acquired using a Zeiss Axioskop 40 microscope equipped with Zeiss AxioCAM image capture system.

\section{IL-17 quantification}

Mouse IL-17 Quantikine ELISA kit (R\&D, M1700) was used to quantify IL-17 produced by BMOL cells transfected with plasmid pUNO1mIL17A in culture supernatant before allograft, and in mouse sera after allograft. The procedure was performed according to the protocol described in the manual.

\section{Transfection of miR-122-mimic}

BMOL cells were prepared in 6-well plates for microRNA (miRNA)-mimic transfection. Twenty-five $\mathrm{nM}$ of hs-miR122-mimic (Dharmacon) was prepared with DharamaFECT1 reagent (Dharmacon), according to manufacturer's instructions and cells were lysed 48 hours after miR-122 transfection for RNA analysis.

\section{Small and mRNA library preparation and sequencing}

Using the TruSeq Small RNA Sample Prep Kit (Illumina), total RNA, containing small RNAs, was reverse transcribed into a cDNA library. In brief, $1 \mu \mathrm{g}$ of total RNA per sample was ligated with $5^{\prime}$ and $3^{\prime}$ adapters, reverse transcribed, treated with RNase, and amplified with PCR using specific labeled amplification primers. To select the size of small RNAs, samples were migrated on $6 \%$ native polyacrylamide gels. To collect miRNA populations, cDNA fragments between 145 and 160 bp were cut from the gel, eluted and precipitated. Finally, cDNA chip was dried and suspended in $10 \mu \mathrm{L}$ of nuclease free water. In parallel, cDNA library was built from total RNA ( $1 \mu \mathrm{g}$ per sample) from the same samples. Purification and fragmentation were performed using the TruSeq RNA Sample Preparation Kit v2 - Set A 
(Illumina). Next, cDNA samples were end repaired using End Repair Mix reagent (Illumina) and double-stranded cDNAs were purified and enriched to create the cDNA library. The quality of cDNA in each final miRNA and mRNA libraries was assessed using the Agilent 4200 TapeStation System (Agilent) and the amount was verified using Quant-iT PicoGreen dsDNA Assay Kit (Invitrogen). Equimolar concentrations of each final library were pooled at a final concentration of $2 \mathrm{nM}$ of cDNA. Libraries were run on the Illumina NextSeq 500 platform using the High Output kit v2 (75 cycles, Illumina).

\section{Bioinformatics analyses of microRNA data}

Quality control of sequence reads generated from Illumina-based next-generation sequencing was assessed using Fastqc (v0.11.5) and trimmed reads (trimmomatic v0.36) were aligned to the mouse reference miRNAs (miRBase v21) of the latest mouse genome assembly by using Bowtie (v2.0.6) to identify known miRNAs. Differences in normalized expression levels of miRNAs in IL-17-treated LPC compared to untreated cells were carried out by the DESeq2 $\mathrm{R}$ package (Bioconductor) using a model based on the negative binomial distribution. P-values were adjusted for multiple testing using the Benjamini-Hochberg method for controlling the false discovery rate (FDR). MiRNAs with a corrected p-value $<0.05$ and fold changes either $>1.5$ or $<0.67$ were assigned as differentially expressed.

\section{Statistical analysis}

Results are expressed as mean \pm S.E.M., and statistical significance was determined by a two-tailed Student's $t$ test or Mann and Whitney $U$ test as appropriate using PRISM 5.0 software. Data were considered significantly different for ${ }^{*} p<0.05$, ${ }^{* *} p<$ $0.01,{ }^{* * *} \mathrm{p}<0.001$.

\section{Results}

\section{The expression of the CSC marker CD133 is correlated with the LPC marker CK19 in human hepatocellular carcinoma}

In order to determine whether CSCs could originate from normal liver progenitor cells (LPC) and their involvement in liver carcinogenesis, we used the public cBioPortal site for web-based analysis of Liver Hepatocellular Carcinoma (TCGA PanCancer Atlas) and Spearman's correlation coefficient to assess the relationship between the mRNA expression levels of the classically used cancer stem cell marker CD133 (alias PROM1) and other genes [23, 24]. The Figure 1 represents a web-based analysis from the Cancer Genome Atlas using 366 HCC samples. The correlation analysis showed that CD133 displayed the highest positive correlation with the expression of CK19 (alias KRT19) which constituted the main
A

\begin{tabular}{|c|c|c|}
\hline Correlated Gene & $\begin{array}{c}\text { Spearman's } \\
\text { Correlation }\end{array}$ & p-Value \\
\hline Krt19 & 0,702 & $1,57 \mathrm{E}-55$ \\
\hline Slc34a2 & 0,652 & $1,03 \mathrm{E}-45$ \\
\hline Gprin2 & 0,640 & $1,45 \mathrm{E}-43$ \\
\hline Mmp7 & 0,637 & $5,08 \mathrm{E}-43$ \\
\hline Wnt7b & 0,630 & $7,96 \mathrm{E}-42$ \\
\hline Sfrp5 & 0,622 & $1,30 \mathrm{E}-40$ \\
\hline Lypd6b & 0,604 & $1,01 \mathrm{E}-37$ \\
\hline Vangl2 & 0,601 & $2,40 \mathrm{E}-37$ \\
\hline Vtcn1 & 0,600 & $4,28 \mathrm{E}-37$ \\
\hline Fras1 & 0,598 & $6,71 \mathrm{E}-37$ \\
\hline Wnk2 & 0,597 & $1,17 \mathrm{E}-36$ \\
\hline Tmem51 & 0,592 & $4,68 \mathrm{E}-36$ \\
\hline Syt13 & 0,591 & $8,35 \mathrm{E}-36$ \\
\hline Clic6 & 0,590 & $1,02 \mathrm{E}-35$ \\
\hline Ephb3 & 0,587 & $2,77 \mathrm{E}-35$ \\
\hline Esrp1 & 0,581 & $1,85 \mathrm{E}-34$ \\
\hline Cftr & 0,579 & $3,26 \mathrm{E}-34$ \\
\hline Spint1 & 0,577 & $6,17 \mathrm{E}-34$ \\
\hline Itgb8 & 0,576 & $1,01 \mathrm{E}-33$ \\
\hline Col9a2 & 0,574 & $1,81 \mathrm{E}-33$ \\
\hline Sctr & 0,571 & $4,14 \mathrm{E}-33$ \\
\hline
\end{tabular}

B

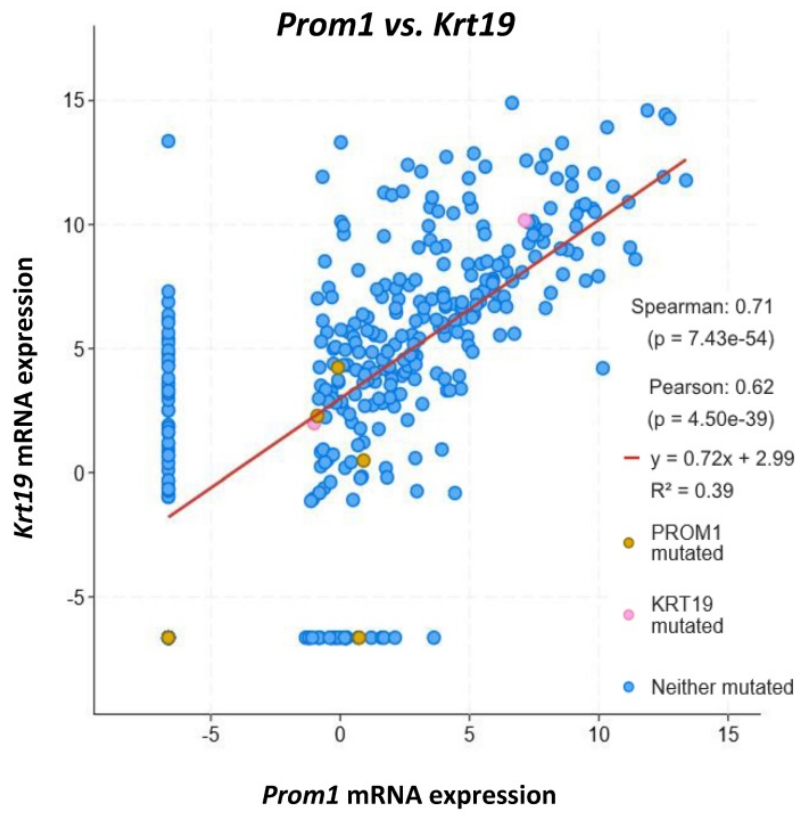

Figure 1. The expression of the CSC marker CD133 is correlated with the LPC marker CK19 in human hepatocellular carcinoma. (A) Visualization of top 20 genes that are correlated with Cdl33 known as Proml mRNA expression with the highest correlation with Ck19 known as Krtl9 (RSEM, batch normalized from Illumina Highseq_RNASeqV2; n=366 HCC samples). (B) Correlation between Proml and Krtl9 mRNA expressions (RSEM, batch normalized from Illumina HiSeqV2) represented with Log scaled axis using a linear regression analysis. 
marker of LPC/biliary cells (Figure $\mathbf{1 A}$ and B). This result suggests a possible origin of CSCs from LPCs in human HCC.

\section{IL-17-producing cells and CSCs are closely located in human cirrhotic livers}

In human cirrhotic livers, we previously reported a correlation between the number of IL-17-producing cells and the expansion of CK19+ biliary and progenitor cells [17]. In order to assess whether an increased and sustained IL-17 production contributes to triggering LPC transformation into CSCs, the expression of IL-17, CK19 and CD133 were assessed by immunohistochemistry on serial sections of cirrhotic livers from various etiologies [17] (Figure 2A) organized in two groups of patients, based on the number of $\mathrm{IL}-17^{+}$cells infiltrating the liver: IL-17Low and IL-17High. In both groups, IL-17-producing cells were located within ductular reactions in close vicinity of $\mathrm{CK} 19^{+} \mathrm{LPCs}$ and $\mathrm{CD} 133^{+} \mathrm{CSC}$. In addition, the numbers of $\mathrm{CK} 19^{+}$and $\mathrm{CD} 133^{+}$cells increased with the number of IL-17-producing cells infiltrating the liver (Figure 2B). Semi-quantitative analysis showed a positive correlation between the number of infiltrated $\mathrm{IL}-17^{+}$cells and CSC accumulation. In the group of patients with low levels of IL-17-infiltrating cells defined as IL-17Low patients, 38\% of cases were expressing high levels of CD133. Contrastingly, among patients displaying high levels of
IL-17-infiltrating cells (IL-17High patients), $75 \%$ of cases highly expressed CD133 marker $(\mathrm{p}<0.05)$. Collectively, these findings reveal an association between the increased number of IL-17-producing cells and the accumulation of CSCs in human cirrhotic livers.

\section{Long-term stimulation of LPCs by IL-17 triggers their malignant transformation into a CSC phenotype}

Long-term stimulation of LPCs by IL-17 enhances their expression of cancer and stemness markers

To investigate potential effects of IL-17 on LPC transformation in vitro, a non-tumor murine LPC line (BMOL) was cultured for long-term $(10,20,30$ or 40 days) with or without IL-17. In those conditions, mRNA expression of CSC (Cd133, Epcam and Aldh), of pluripotency (Klf4) and of tumor cell (Gpc3 and Afp) markers were found significantly induced by IL-17 when compared to those from control non-treated LPCs (Figure 3A). Furthermore, flow cytometry analysis showed that 8 to $10 \%$ of IL-17-treated LPCs acquired CD133 protein expression after 10, 20 or 30 days but not in non-treated LPCs (Figure 3B). Similar neo-expression of CSC-related genes induced by IL-17 was evidenced in human hepatic progenitor cell line HepaRG (Supplemental Figure 1). In this model, sustained IL-17 treatment reduced the mRNA
A
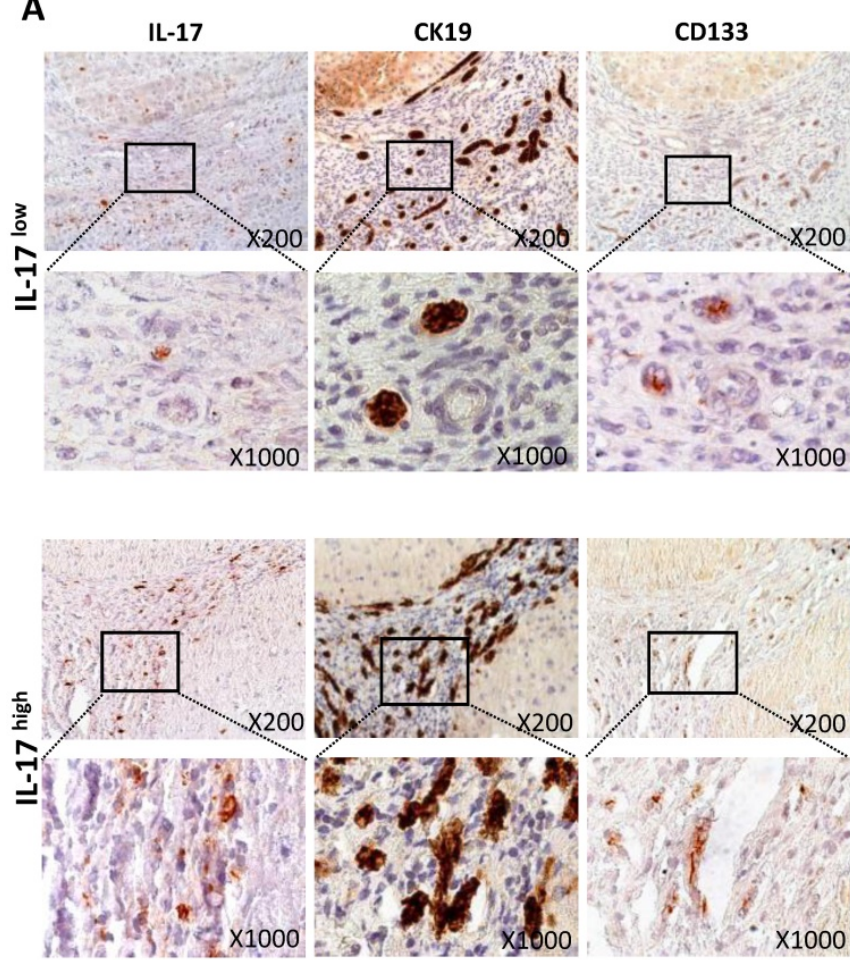

B

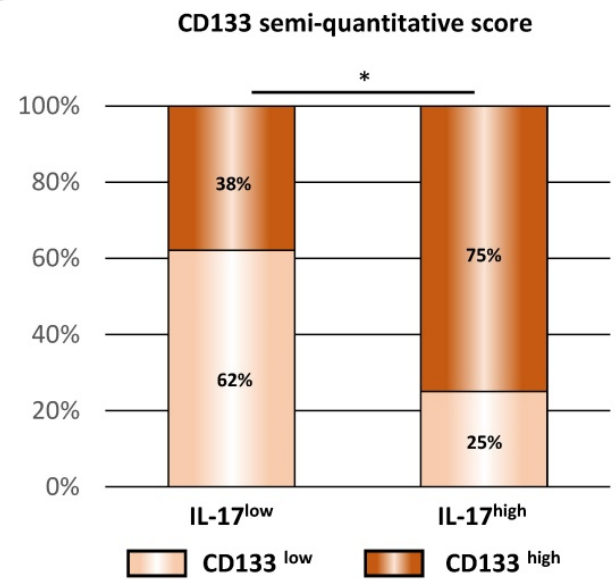

Figure 2. IL-17-producing cells and CSCs are closely located in human cirrhotic livers. (A) Interleukin-17A (IL-17), cytokeratin-19 (CK19) and CD133 immunostainings on serial sections of preneoplastic hepatocellular lesions in human livers. (B) Relative CD133 immunolabelling was quantified in the two groups of patients with high $(n=16)$ versus low $(n=29)$ IL-17 expression, and statistical differences between the groups were assessed with a Chi-square test. 
expression of the two hepatocytic markers $A l b$ and $H n f 4 \alpha$ (Supplemental Figure 1A), while inducing mRNA expression of stem cell markers such as $C d 133$ and Epcam (Supplemental Figure 1B). These results strongly suggest that IL-17 promotes the transformation of both murine and human LPCs into a CSC phenotype.
A

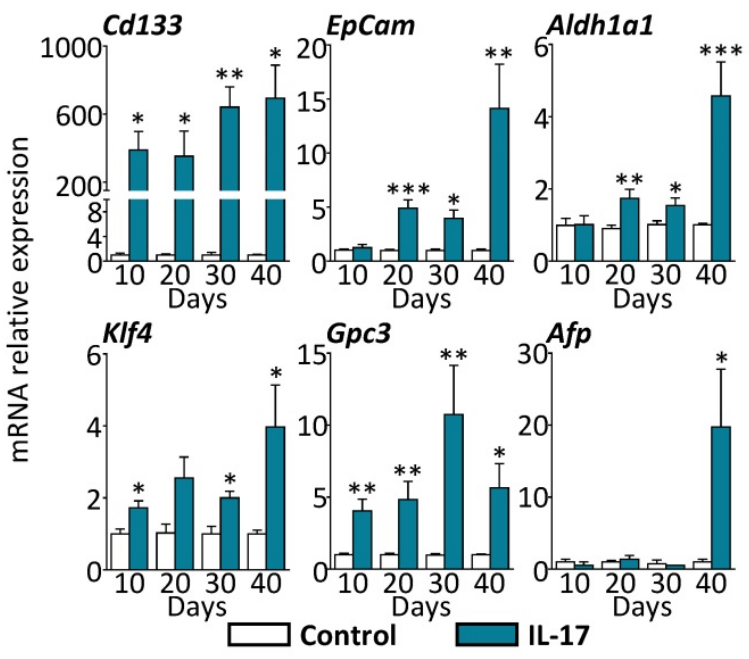

C

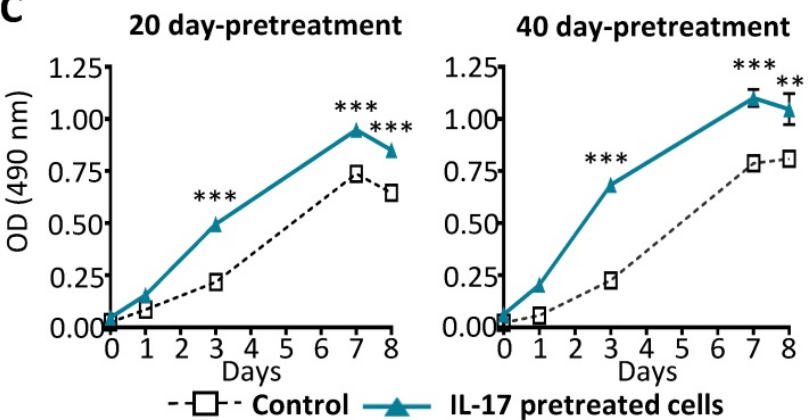

B

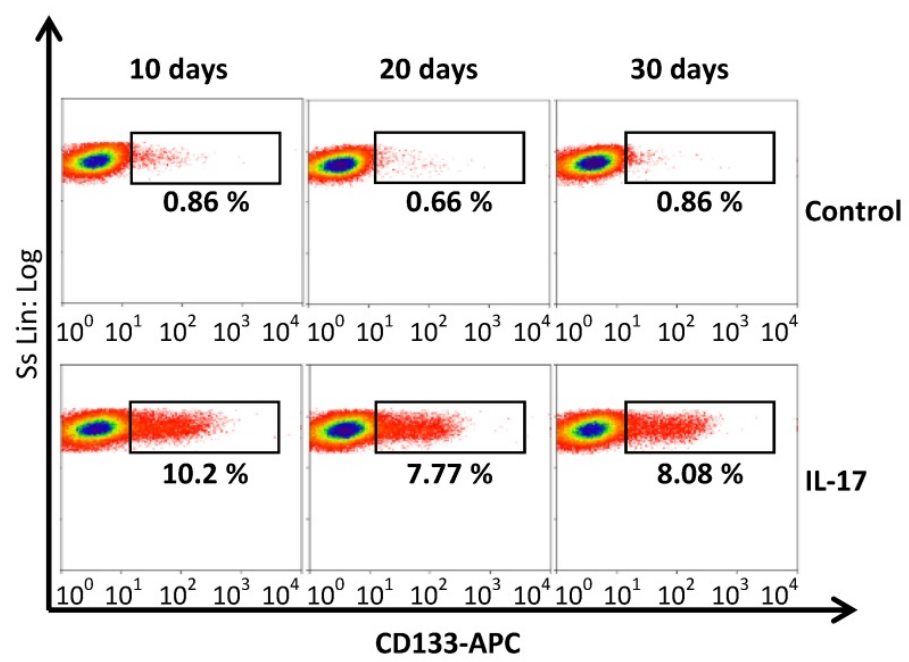

D

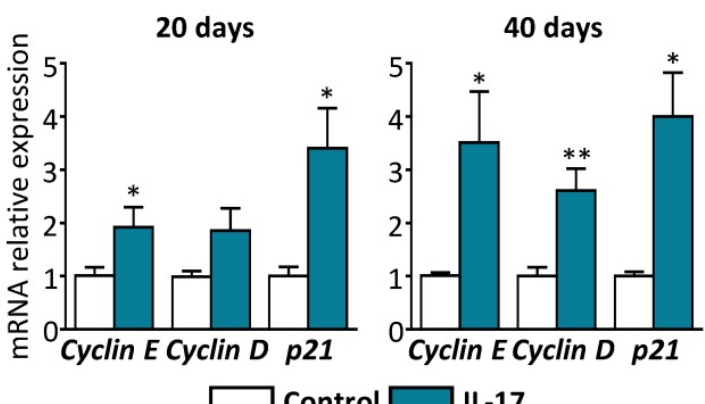

$\mathbf{E}$

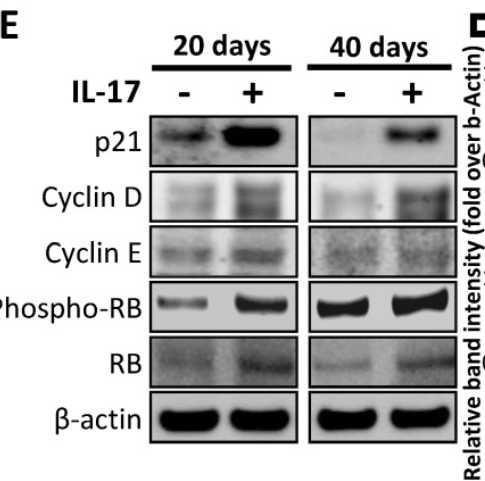

$\mathbf{G}$

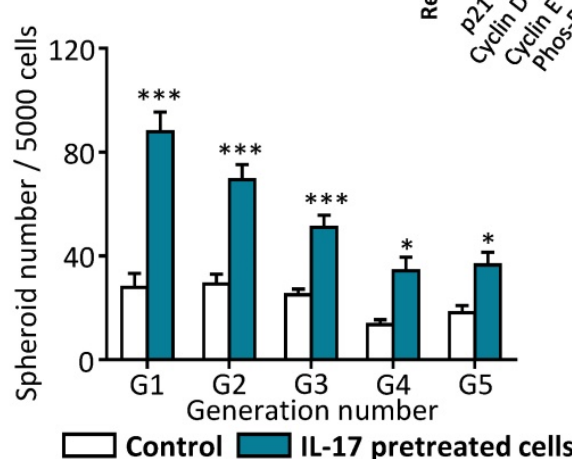

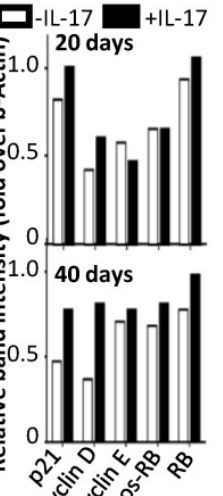

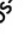

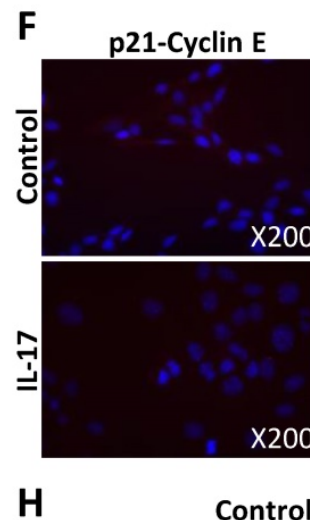

Control p21-Cyclin D

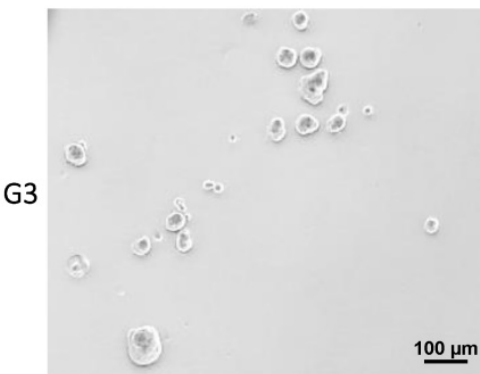

IL-17 pretreated cells

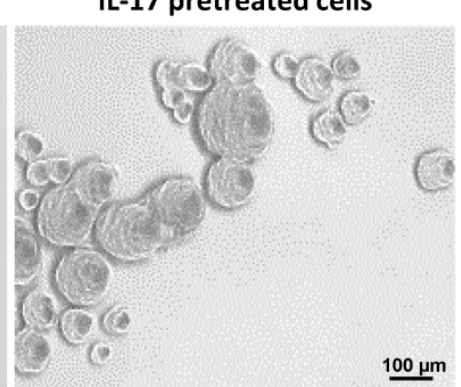

Figure 3. Long-term stimulation of LPCs by IL-17 triggers their malignant transformation into a CSC phenotype. (A) LPC were stimulated for 10 to 40 days with IL-17 and relative mRNA expression of cancer (Gpc3 and Afp) and stem cell markers (CdI 33, Epcam, Aldh, KIf4) were analyzed by RT-qPCR and expressed as fold change over 
untreated cells (control). (B) CD133-positive LPCs were quantified by flow cytometry analysis after IL-17 treatment for 10 to 30 days. (C) After pretreatment with IL-17 for 20 and 40 days, LPC proliferation rate was assessed every day for 8 days using MTS test. Data are representative of 3 independent experiments. (D) Fold change in Cyclin DI, Cyclin $E$ and P2I mRNA expression was determined by relative QPCR analysis of LPC treated with IL-17 or vehicle (control) for 20 or 40 days. (E) Western blot analysis of cell cycle related-proteins P21, cyclin D and, E and total retinoblastoma (RB) or phosphorylated retinoblastoma protein (phospho-RB) in LPCs treated or not (control) with IL-17, for 20 or 40 days. The western blot band intensity is quantified by using image $\mathrm{J}$ software. The graphs represent the intensity of each band as ratio over b-actin band intensity. (F) Cyclin $\mathrm{D}$ and E interaction with P21 was assessed by the Duolink Proximity Ligation Assay on LPC treated with IL-17 for 40 days and shown by the presence of red spot (insets with higher magnification). Nuclei were stained with DAPI. Close-proximity of p21 and cyclin D (red) is only observed in IL-17-treated LPC. (G) After 30 days of IL-17 pretreatment or without pretreatment (control), LPC were cultured in sphere formation condition assay over 5 generations and total spheroid number per well (larger than $40 \mu \mathrm{m}$ ) was counted for each generation (from G1 to G5). Experiments were performed in sextuplicate in 3 independent experiments. (H) Representative micrographs show formed spheroids (scale bar $100 \mu \mathrm{m}$ ) at G3 from LPC pretreated (or not, control) with IL-17. Data represent mean \pm SEM for 3 independent experiments performed in triplicate and *P $<0.05$; **P $<0.01$; ***P $<0.001$ for control versus IL-17-treated or pretreated cells.

Long-term stimulation of LPCs by IL-17 enhances their self-renewal properties

In a next step, we proposed to determine whether the acquisition of IL-17-induced CSC marker expression by LPCs was associated with enhanced cell cycle activity and self-renewal capacities. For that purpose, an MTS cell proliferation assay was performed on BMOL cells pretreated with IL-17 for 20 or 40 days. Then, the proliferation rate was measured every day for 8 days of culture without adding IL-17 in the medium. Under these conditions, IL-17-pretreated cells for either 20 or 40 days, had acquired enhanced cell cycle activity when compared to non-pretreated control cells (Figure 3C). This effect was associated with a significant increase in Cyclin D1, Cyclin E and p21 (CDKN1A/waf1) at mRNA (Figure 3D) and at protein levels (Figure 3E) in BMOL cells pretreated with IL-17 for 20 or 40 days. The protein levels quantified by image $\mathrm{J}$ showed an increase in P21, Cyclin D, E and Retinoblastoma expression at 20 and 40 days under IL-17 stimulation (Figure 3E). Depending on which partner p21 is interacting with, its impact on cell cycle regulation will be different. The interaction of p21 with Cyclin E prevents cell-cycle progression by inhibiting the activity of cyclin E/CDK2 complexes. In contrast, p21 interaction with Cyclin D1 results in retinoblastoma (RB) hyperphosphorylation and activation of mitogenic pathway [25]. To identify p21 partner in LPCs treated with IL-17, a PCR Ligation-Assay (PLA) was performed. A close proximity of p21 and cyclin D was evidenced by increased fluorescent dots in condition where LPCs are treated with IL-17 and no interaction between p21 and cyclin E was observed (Figure 3F). Furthermore, a 5-generation spheroid formation assay was performed in vitro as a functional test assessing the self-renewal capacity of these cells. Only spheroids with diameters higher than $40 \mu \mathrm{m}$ were counted. We showed that LPCs pretreated with IL-17 for 30 days had acquired self-renewal properties as compared to non-pretreated cells from the first generation (Figure 3G). This effect was maintained for 5 generations and illustrated by a microscopic photography taken at the third generation (Figure $3 \mathbf{H})$. These results indicate that IL-17 pretreatment enhances LPCs self-renewal capacity, and this effect is maintained over generations. Taken together, these findings demonstrated that long-lasting IL-17 pretreatment of LPCs leads to their conversion into cells with a CSC phenotype.

\section{IL-17 induces LPC transformation into CSCs through miR-122 down-regulation}

Several pieces of evidence suggest that miRNA have regulatory functions in cancer initiation, and dysregulation of miRNAs occurs frequently in a variety of liver diseases, including HCC [26]. To determine the underlying mechanism by which IL-17 favors the expansion of CSCs and subsequent tumor initiation and progression, we attempted to identify miRNAs that are dysregulated by IL-17 and could represent a molecular signature of IL-17 effects during hepatocarcinogenesis (Figure 4A). Among the most dysregulated miRNAs, miRNome analysis revealed a decrease in miR-122-5p expression in IL-17-treated LPCs when compared to non-treated LPCs. MiR-122-5p is by far the most abundant miRNA expressed in murine and human hepatocytes that has been shown to be downregulated during hepatocarcinogenesis [27], while miR-465a/b/c-3p expression was found only in murine cells. This finding was validated by RT-qPCR showing a $90 \%$ decrease of miR-122-5p expression in IL-17 treated cells when compared to untreated LPCs (Figure 4B).

To further determine whether miR-122 downregulation could be responsible for LPC transformation, we then transfected LPCs with mir-122 mimic in vitro (Figure 4C). Efficiency of transfection was validated by qPCR showing a strong elevation of miR-122 expression in both IL-17-treated or not (control) transfected-LPCs (Supplemental Figure 2). Overexpression of miR-122 in LPCs abolished self-renewal capacity acquired by IL-17 pretreatment. The number of spheroids obtained from IL-17-pretreated LPCs was higher than the spheroid number formed from untreated LPCs. However, overexpression of miR-122 mimic was sufficient to abolish IL-17-induced self-renewal capacities (Figure 4D and E). In addition, miR-122 mimic overexpression restored albumin expression while reducing the expression of stemness markers such as Aldh1a1 and of cell cycle related-genes including Cyclin D, E and Pcna (Figure 4F). 


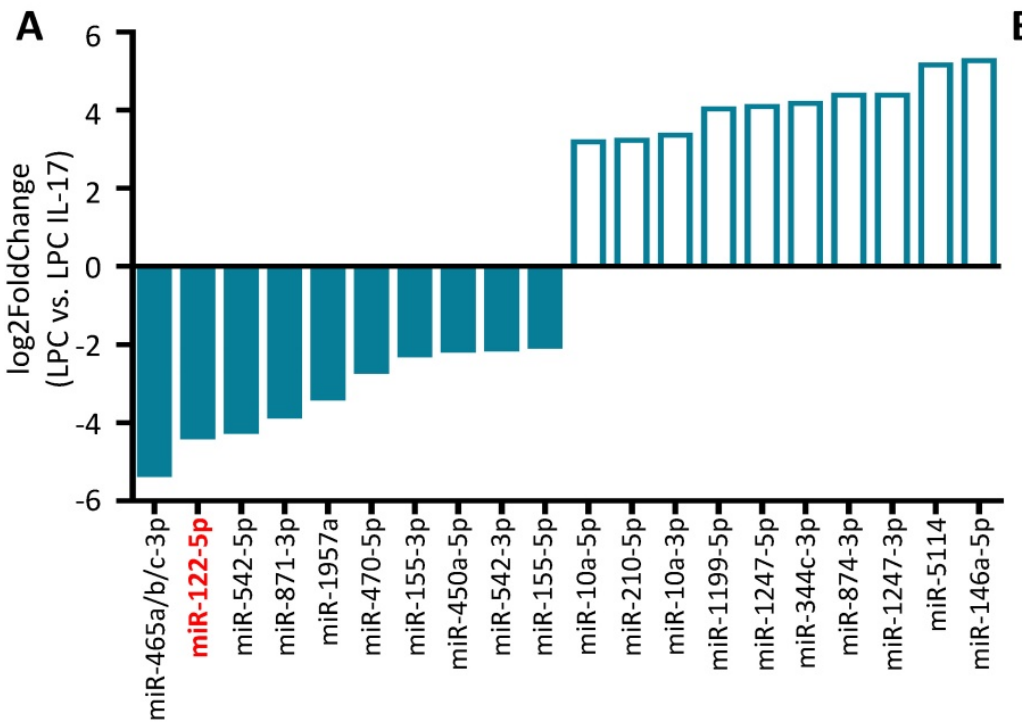

B

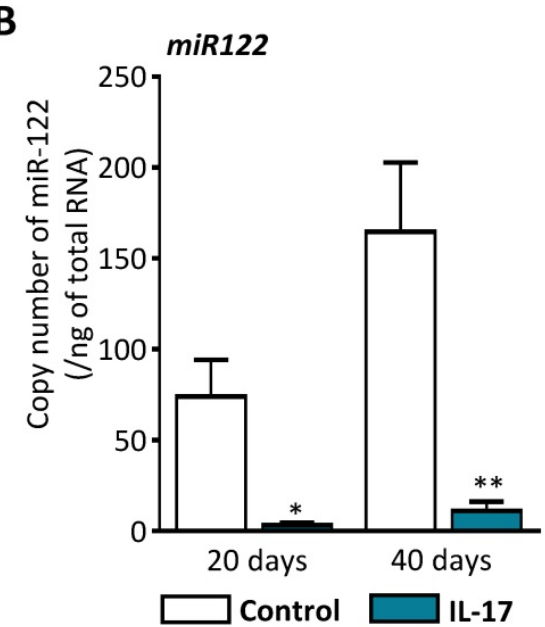

C

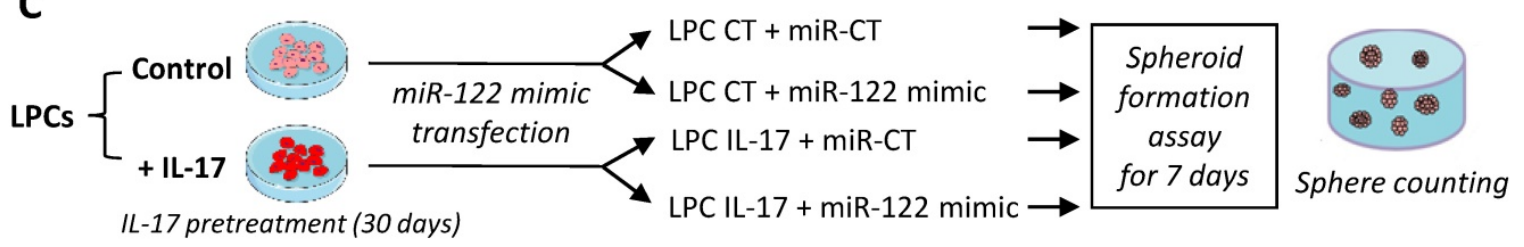

D

miR-CT

miR-122 mimic

E
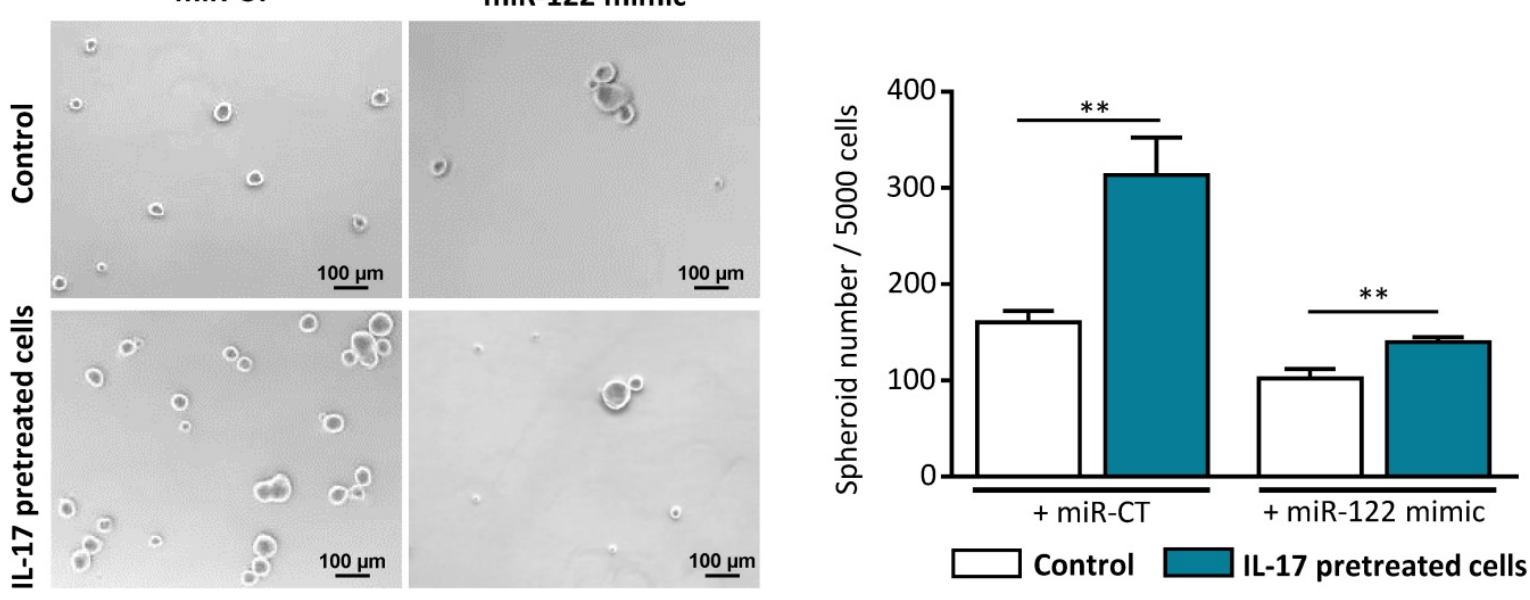

$\mathbf{F}$
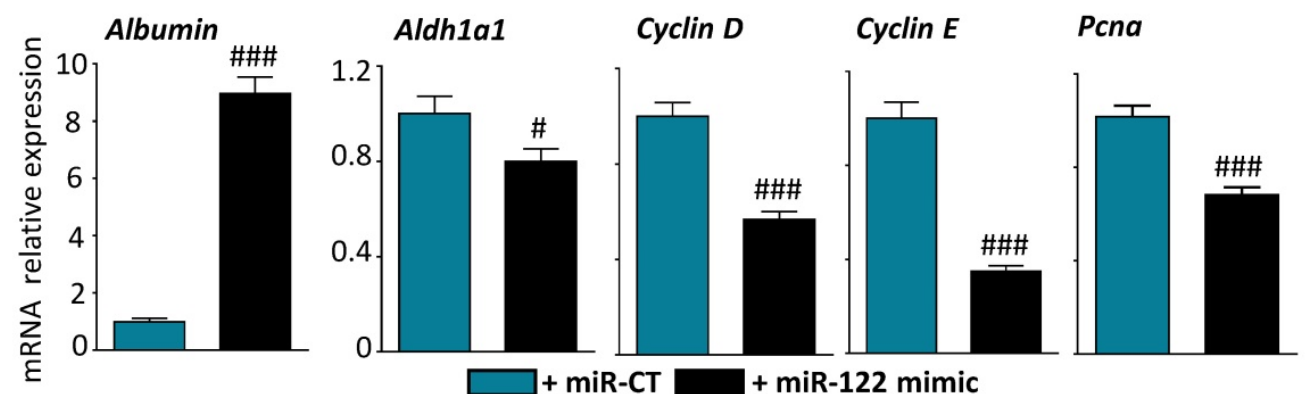

Figure 4. IL-17 induces LPC transformation into CSCs through miR-122 downregulation. (A) Top 10 up (white bars) and down (blue bars) of the most dysregulated microRNAs in IL-17-treated LPC compared to untreated cells after 40 days of culture. (B) Expression of miR-122 was assessed by qRT-PCR in IL-17-treated or not (control) LPC for 20 or 40 days. (C) Control (LPC CT) or IL-17-pretreated LPC for 30 days (LPC IL-17) were transfected with either a control miRNA (mir-CT) or miR-122 mimic and (D) next cultured in sphere formation assay for 7 days $($ scale bar $100 \mu \mathrm{m})$. (E) Representative micrographs showed number of formed spheroids with a diameter greater than $50 \mu \mathrm{m}$ (F) Messenger RNA expressions of Albumin, Aldh IAI and cell-cycle markers (Cyclin D, E, Pcna) were assessed by qRT-PCR in IL-17 pretreated LPC for 30 days and transfected either with miR-CT or miR-122 mimic. Data represent mean \pm SEM, $\mathrm{n}=6$ per condition. $* \mathrm{P}<0.05 ; * * \mathrm{P}<0.01$ for control versus IL-17 treated or pretreated cells; \#P<0.05 and \#\# < 0.001 for miR-CT treated cells versus miR-122 mimic-treated cells. 
A

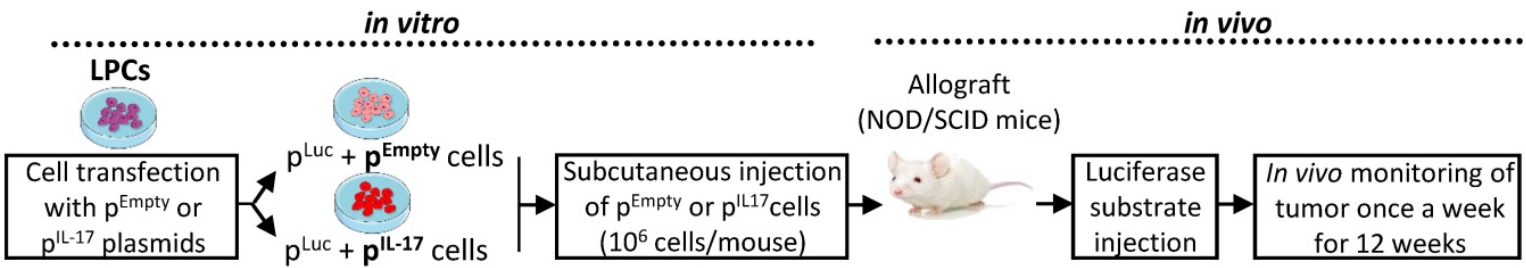

B

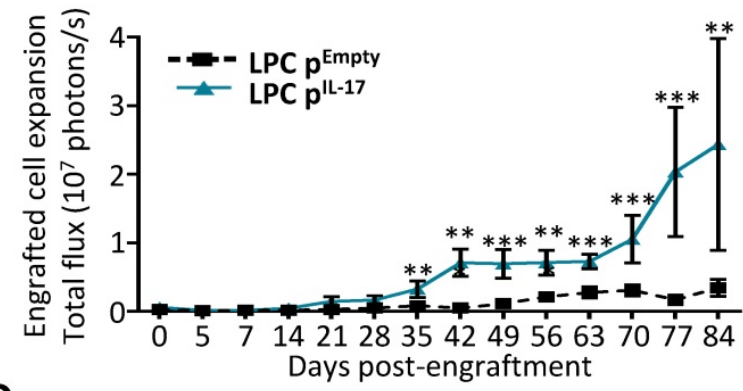

C

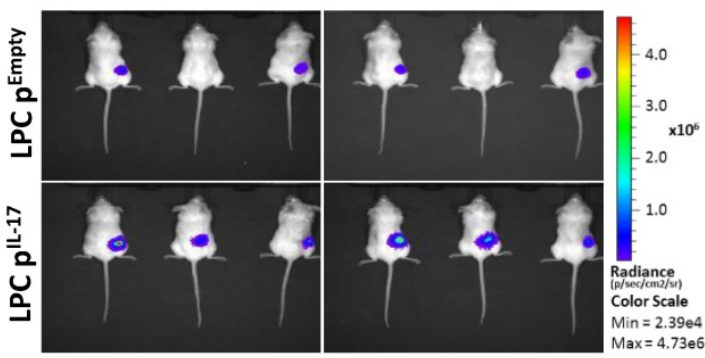

D

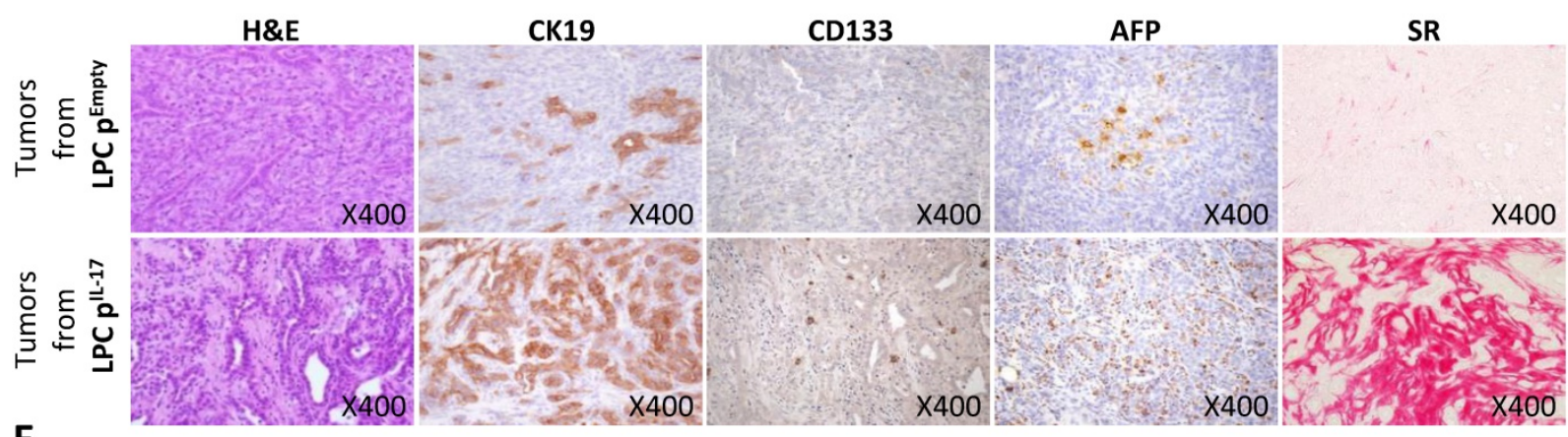

E

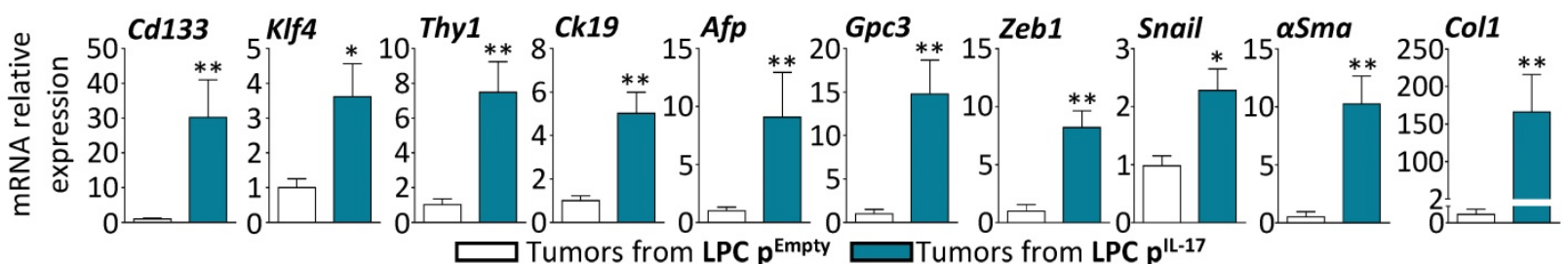

Figure 5. Constitutive expression of IL-17 in LPCs enhances tumor expansion with an aggressive phenotype in vivo. (A) NOD/SCID mice were inoculated

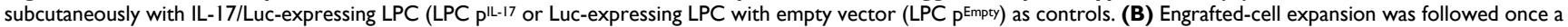
week for 84 days by in vivo bioluminescence imaging. (C) Representative average radiance (photons/s per square centimeter per steradian) of tumor bearing mice 56 days after cell engraftment according to the associated color scale. (D) Histological and immuno-histochemical analyses of tumors 84 days after LPC inoculation was performed. (E) Messenger RNA expression of CSC (CD133, KLF4, Thy-1, CK-19, AFP and GPC3), and EMT/fibrotic (Zeb-1, Snail, $\alpha$-SMA and Col-1) markers were assessed by RT-qPCR in isolated tumors at 84 days. Data represent mean \pm SEM, $n=6$ mice per group and $* P<0.05 ; * * P<0.01 ; * * * P<0.001$ for control LPC $P^{\text {Empty }}$ versus $P^{\text {il-17 }}$ engrafted mice.

\section{Constitutive expression of IL-17 in LPCs enhances tumor expansion with an aggressive phenotype in vivo}

To further characterize IL-17 tumorigenic effect in vivo, an LPC line highly expressing IL-17 was generated using a stable co-transfection with mouse IL-17 plasmid (LPC $\mathrm{p}^{\mathrm{IL} 17}$ ) or an empty vector as control (LPC $p^{\text {Empty }}$ ), combined with pGL4.51 (Luc2) vector in BMOL cells. Transfected cell phenotype was verified prior to in vivo engraftment and the results showed that IL-17 constitutive expression up-regulated cancer cell and CSC markers (Gpc3 and Cd133) as compared to control cells (Supplemental Figure $3 \mathbf{A}, \mathbf{B})$. In addition, IL-17 production by transfected-LPCs was verified in the culture medium by ELISA before engraftment (Supplemental Figure 3C). Next, LPC $p^{\text {IL17 }}$ or LPC $p^{\text {Empty }}$ cells were subcutaneously engrafted into immunodeficient NOD/SCID mice. Their expansion was subsequently monitored for 12 weeks by bioluminescence imaging (Figure 5A). Engrafted LPC $\mathrm{p}^{\mathrm{IL} 17}$ cells significantly expanded, whereas LPC $p^{\text {Empty }}$ cells did not (Figure 5B). A representative figure illustrates the higher- 
level of bioluminescence reflecting the number of expanded cells, recorded at day 84 in mice engrafted with LPC $\mathrm{p}^{\mathrm{IL} 17}$ when compared to those engrafted with LPC $\mathrm{p}^{\text {Empty }}$ cells (Figure 5C). Histological analysis of the tumors after an H\&E staining revealed a typical morphological aspect of mixed HCC and cholangiocarcinoma in IL-17-enriched tumors, but not in tissues obtained from LPC $\mathrm{p}^{\text {Empty }}$-engrafted cells (Figure 5D). The tumor phenotype obtained from LPC $\mathrm{p}^{\mathrm{IL17}}$-engrafted cells was associated with a greater expression of the CK19 biliary/progenitor cell marker, and an increased number of $\mathrm{CD}_{133^{+}}$and $\mathrm{AFP}^{+}$cells. This was also associated with increased fibrosis as revealed by Sirius red (SR) staining.

In order to characterize the tumors deriving from LPC $\mathrm{p}^{\mathrm{IL} 17}$ cells, an extraction of RNA from tumor tissues from both engrafted mice was performed. The obtained results showed that tumors from LPC $\mathrm{p}^{\mathrm{IL}-17}$ have a significant increase in cancer cell and CSC markers (Cd133, Klf4, Thy1, Ck19, Afp and Gpc3) when compared to tumors from LPC $\mathrm{p}^{\text {Empty }}$ (Figure 5E). A significantly increased expression of EpithelialMesenchymal Transition (EMT)-related genes (e.g. Snail and Zeb1) and of fibrosis-related genes (e.g. aSma and Col1) was also observed in LPC pIL17-derived tumors. Collectively, these data revealed that an IL-17-enriched microenvironment confers an aggressive phenotype to LPC-derived tumors with histopathological features of mixed HCC and cholangiocarcinoma phenotype.

\section{Constitutive production of IL-17 triggers stem-like phenotype transformation of LPCs, initiates liver fibrogenesis and downregulates hepatic miR-1 22 expression}

To determine the impact of systemic IL-17 on liver, serum IL-17 levels in LPC $p^{\text {Empty }}$ and in LPC $p^{\mathrm{IL}-17}$ engrafted-mice were measured 12 weeks after allograft (Figure 6A). Serum IL-17 levels reached $202 \pm 34 \mathrm{pg} / \mathrm{mL}$, a range comparable to that observed in cirrhotic and HCC patients [28]. Histological examination of livers from LPC $\mathrm{p}^{\text {Empty }}$ or $\mathrm{p}^{\mathrm{IL}-17}$ engrafted mice after 12 weeks, did not reveal any difference in hepatocyte morphology or liver architecture (Figure 6B). In contrast, constitutive IL-17 release in the blood increased hepatic CK19 and CD133 immunostaining, and sinusoidal fibrogenesis as revealed by Sirius Red (SR) staining, in the livers from LPC $\mathrm{p}^{\mathrm{IL}-17}$-engrafted mice (Figure 6B). Moreover, RT-qPCR analysis showed enhanced expression of HCC (Afp, Gpc3), CSC (Aldh1a1, Cd133, Cd44, Klf4, Lgr5, Thy1), EMT (Snail, Zeb1) and fibrosis (aSma) related-genes in the livers from LPC $\mathrm{p}^{\mathrm{IL}-17}$-engrafted mice (Figure 6C). In order to determine whether IL-17 impacts miR-122 expression in the liver, an extraction of total RNAs including small RNAs from livers of
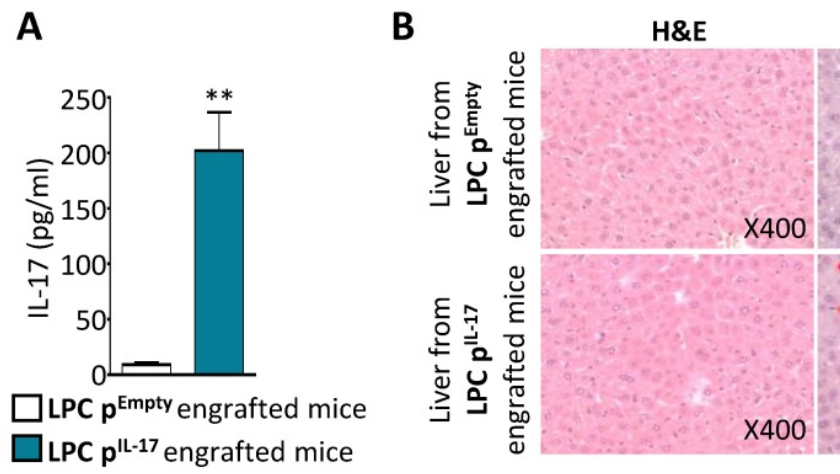

CK19

CD133
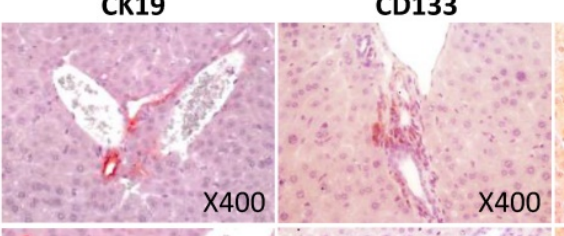

SR

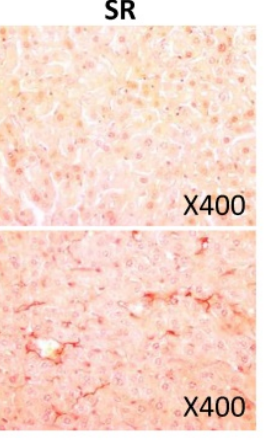

C
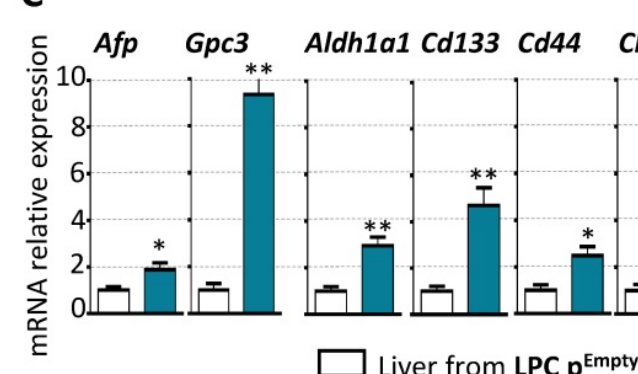

Ck19

KIf4

Lgr5

Thy1

Sna

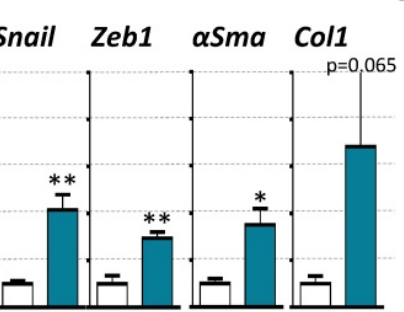

D

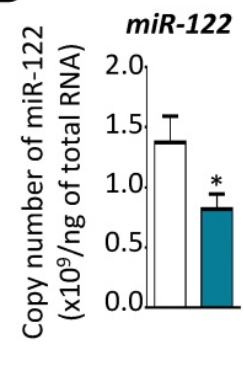

Figure 6. Constitutive production of IL-17 trigger stem-like phenotype transformation of LPCs, initiates liver fibrogenesis and downregulates hepatic

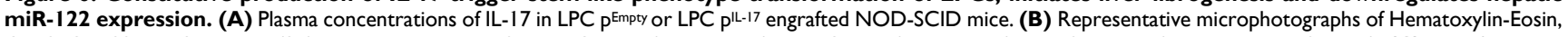

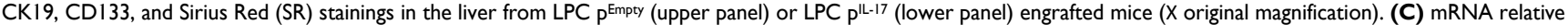
expression analysis of tumor (Afp, Gpc3), CSC (Aldhlal, Cdl33, Cd44, Klf4, Lgr5, Thyl), fibrotic ( $\alpha S m a, C k 19$ Coll) and EMT (Snail, Zebl) markers by qPCR in the liver from LPC $P^{\text {Empty }}$ or LPC $P^{\mathrm{LL}-17}$ engrafted mice. $* P<0.05$, **P<0.01: liver from LPC $P^{\text {Empty }}$ vs LPC $P^{\mathrm{IL}-17}$ engrafted mice. (D) Total copy number of miR-122 per ng of total RNA assessed by $q P C R$ in the liver from LPC $\mathrm{P}^{\mathrm{Empty}}$ or LPC $\mathrm{P}^{\mathrm{IL}-17}$ engrafted mice. ${ }^{*} \mathrm{P}<0.05$; $* * \mathrm{P}<0.01$ : liver from LPC $\mathrm{P}^{\mathrm{Empty}} \mathrm{vs} \mathrm{LPC} \mathrm{p}^{\mathrm{IL}-17}$ engrafted mice. Data represent mean \pm SEM, $\mathrm{n}=6 \mathrm{mice}$ per group. 
LPC $\mathrm{p}^{\text {Empty }}$ or $\mathrm{p}^{\mathrm{IL}-17-e n g r a f t e d}$ mice was performed. Interestingly, analysis of miR-122 expression by qPCR revealed that IL-17 sustained production significantly decreased miR-122 expression in livers from LPC $\mathrm{p}^{\mathrm{IL}-17-e n g r a f t e d}$ mice compared to LPC $\mathrm{p}^{\text {Empty- }}$ engrafted animals (Figure 6D). Together, these results indicate that long-term systemic IL-17 production induces stem-like phenotype transformation of LPCs, favors liver fibrogenesis and downregulates hepatic miR-122 expression.

\section{IL-17-neutralizing strategies reduce tumor growth by limiting CSC occurrence and preventing miR-122 downregulation in vivo}

To understand how IL-17 impacts tumorigenesis in the liver, tumor formation was measured in vivo using a murine model of HCC induced in a fibrotic context by $\mathrm{DEN}+\mathrm{CCl}_{4}$ administration (Figure 7A). Macroscopic analysis of livers from $\mathrm{DEN}+\mathrm{CCl}_{4}$ mice revealed that the number of nodules was lower in the livers of IL-17-deficient mice treated with $\mathrm{DEN}+\mathrm{CCl}_{4}$, as compared to wild-type (WT) treated-mice (Figure 7B, left panel), whereas the liver weight to body weight ratio was similar in the two groups (Figure 7B, right panel). Furthermore, IL-17-deficient mice displayed a significant reduction of hepatic fibrosis (Figure 7C) and tumor areas (Figure 7D). Collectively, these data suggest that IL-17 blockade could reduce the tumor burden in vivo. To verify this hypothesis, WT mice treated with $\mathrm{DEN}+\mathrm{CCl}_{4}$ were intraperitoneally injected twice a week with $100 \mu \mathrm{g}$ of anti-IL-17 neutralizing antibody or control isotype and for 6 weeks (Figure 7E). On macroscopic examination, the livers of anti-IL-17-treated $\mathrm{DEN}+\mathrm{CCl}_{4}$ mice harbored fewer multi-subcapsular nodules of less than $5 \mathrm{~mm}$ than those from isotype control-treated mice, whereas similar liver weight to body weight ratios and fibrosis levels were observed in the 2 groups (Figure $7 \mathbf{F}$ and G). Tumor area quantification using the QuPath software on whole digital H\&E slides showed a significant reduction of the percentage of tumor area in the anti-IL-17-treated mice group, as compared to the control group (Figure 7H). Moreover, IL-17 blockade led to a 2-fold decrease in $\mathrm{AFP}^{+}$(Figure 7I) and $\mathrm{CD}^{3} 3^{+}$(Figure 7J) cells counted in both peri- and intra-tumor areas. Finally, RT-qPCR analysis revealed a strong elevation of miR-122 expression in tumor (Figure 7K) and nontumor parenchyma (data not shown) from $\mathrm{DEN}+\mathrm{CCl}_{4}$ mice that received anti-IL-17 therapy. Together, these results demonstrated that IL-17-deficiency or anti-IL17 therapy led to reduce liver tumor development and allowed to inhibit LPC transformation into CSCs, most likely by restoring miR-122 expression.

\section{Discussion}

The cellular origins and the molecular events that drive primary liver cancer occurrence and growth remain unclear. Recent findings suggest that CSCs can play the role of tumor-initiating cells [6]. One of the questions that needs to be addressed is whether tumors arise from dedifferentiation of epithelial cells (hepatocytes and biliary cells) or from a blockade of LPC differentiation and cell-cycle dysregulations leading to their transformation into CSCs [5].

Clinical observations in patients with chronic liver diseases revealed an increased number of CK19+ LPCs with the severity of the diseases [7, 17] Moreover the presence of stemness markers such as CD133 was associated with a poor prognosis and an aggressive tumor behavior of primary liver cancers [29]. While $\mathrm{CD}_{133}{ }^{+} \mathrm{CSC}$ s were found expressed in up to $40 \%$ of HCC [30], they were identified in the majority of ICC [31], and their presence was associated with poor clinical outcomes.

In experimental animal models, LPCs have been described as stem/progenitor cells contributing to liver carcinogenesis [4]. Remarkably, subcutaneous engraftment of LPCs isolated from cirrhotic or HCC into immunodeficient mice led to tumor development in contrast to LPCs isolated from non-cirrhotic or non-cancerous livers $[13,32]$. Together these findings strongly suggest that LPC transformation into CSCs contributes to the initiation of primary liver cancers. Thus, it is of interest to identify the mechanisms allowing LPC conversion into CSCs [33] to propose suitable strategies preventing cancer initiation and expansion [8].

Primary liver cancers generally develop in a context of chronic inflammation. In that inflammatory context, a recent meta-analysis revealed that IL-17 is associated with a poor prognosis of gastrointestinal tumors [34] and that IL-17 is also considered as a tumor-promoting cytokine that regulates macrophage activation and cholesterol synthesis in hepatocytes in an experimental model of alcohol-induced HCC [35]. In addition to a key role described for this cytokine in tumor progression and in resistance to anticancer therapies, the fact that IL-17 is also produced at preneoplastic stages of chronic inflammatory liver diseases suggests its involvement in tumor initiation. Interestingly, IL-17-mediated activation of stem/ progenitor compartment has been largely described in gastrointestinal [36, 37], skin [38], ovarian [39], pancreatic [40], breast [41] and prostate [42] cancers. Thus, this proinflammatory cytokine could directly act on the stem cell compartment and thereby contribute to the development of a wide range of malignancies [16]. In this study, we showed that IL-17 producing cells are located in close vicinity of CD133+ 
cells and CK19+ LPCs in human cirrhotic livers. We previously demonstrated that IL-17 promotes CK19+ LPC accumulation within ductular reaction [17] and here, we evidenced a positive correlation between the number of infiltrated $\mathrm{IL}^{-17^{+}}$cells and CSC accumulation in preneoplastic human liver areas.
Because CD133 is the main surface marker of CSC populations in various solid tumor types, these results prompted us to hypothesize that in chronic liver diseases, IL-17 could be involved in $\mathrm{CD}_{133^{+}}$cell accumulation as a consequence of a transformation of LPCs into CSCs.

A

IL-17 deficiency in DEN $+\mathrm{CCl}_{4}$ mice

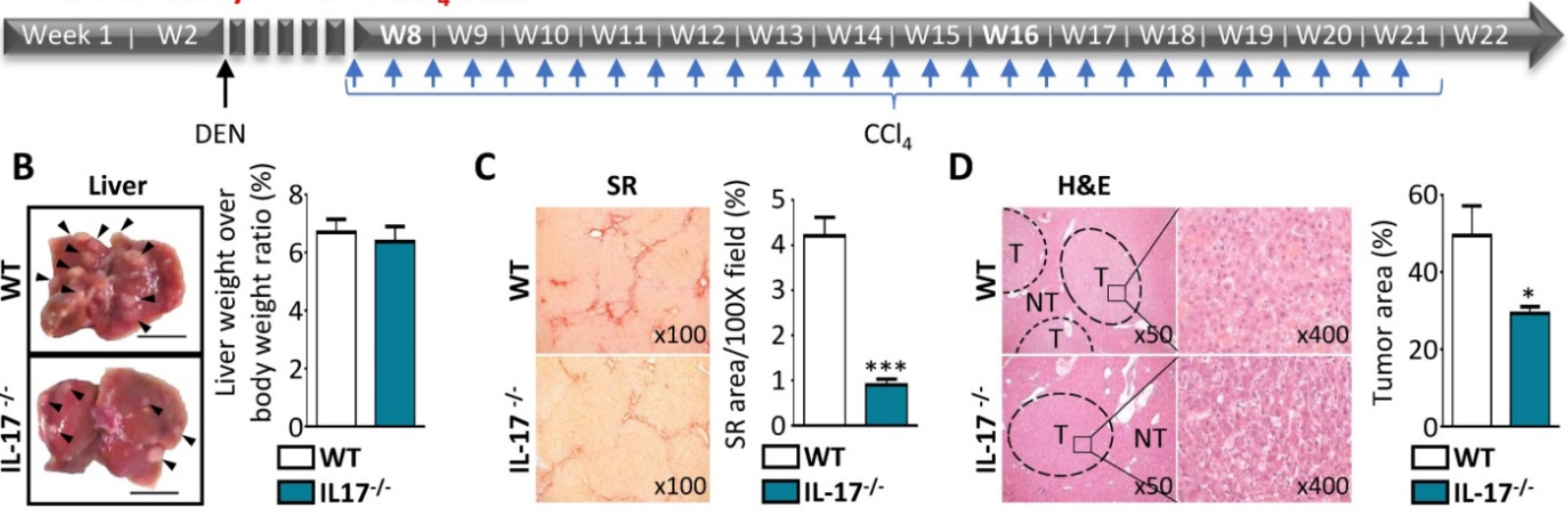

E

Anti-IL-17 therapy in DEN $+\mathrm{CCl}_{4}$ mice

Week 1 | W2 17001 W8 | W9 | W10 | W11 | W12 | W13 | W14 | W15 | W16 | W17 | W18| W19 | W20 | W21 | W22 $\uparrow$

F

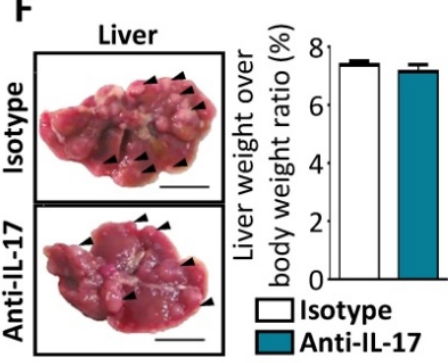

G

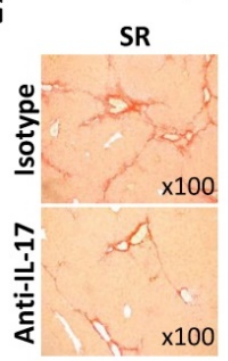

$\mathrm{CCl}_{4}$

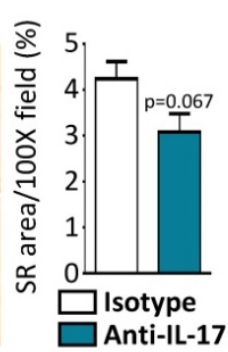

H
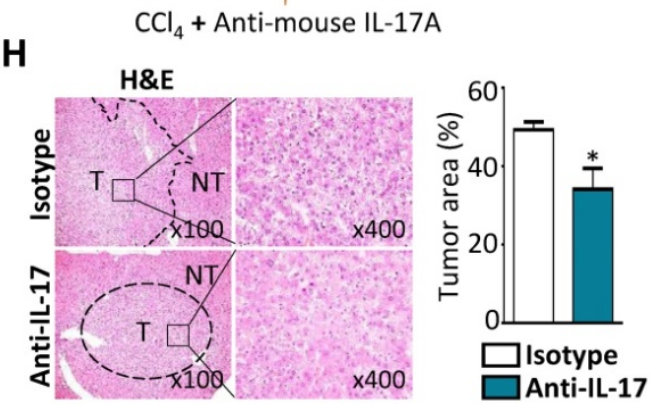
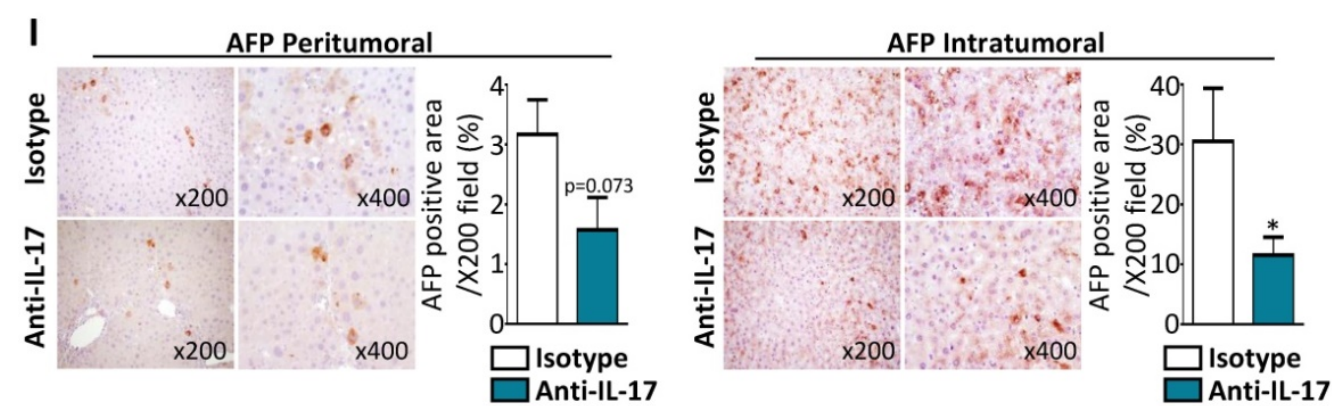

J
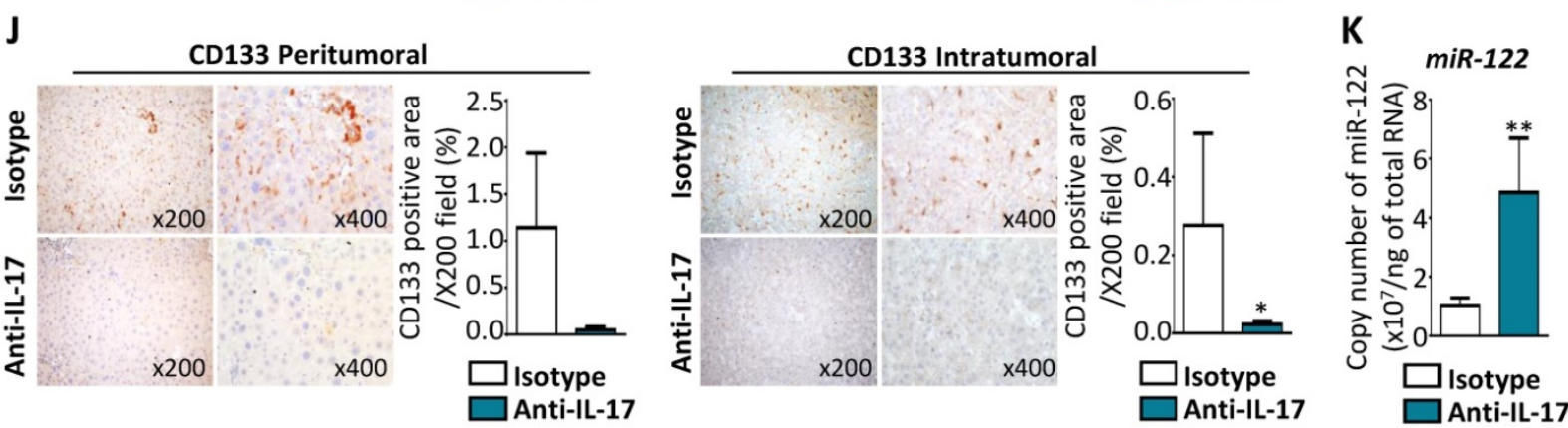

Figure 7. IL-17-neutralizing strategies reduce tumor growth by limiting CSC occurrence and preventing miR-122 downregulation in vivo. (A) Schematic representation of experimental design used for chronic DEN and $\mathrm{CCl}_{4}$ administration in WT or IL-17/- mice. (B) Representative macroscopic liver morphology from 
22-week-old WT or IL-17- mice subjected to the DEN and $\mathrm{CCl}_{4}$ murine model of liver cancer (left panel with scale bars represent 1 mm and arrows show tumor nodules). Quantification of liver weight over body weight ratios (right panel). (C) Representative microphotographs and quantification of Sirius red staining in the liver of WT and IL-17-/mice. (D) Hematoxylin-Eosin staining allows to highlight non-tumor (NT) and tumor (T) liver tissues at two different magnifications (left x500 and right $x 400$ ) and quantification of tumor areas in the liver of WT and invalidated mice (right). (E) Experimental design for anti-IL-17 therapy in DEN+ $\mathrm{CCl}$-induced $\mathrm{HCC}$ model. (F) Representative macroscopic liver morphology (scale bar $1 \mathrm{~mm}$ and arrows show tumor nodules and liver weight over body weight ratios measure in mice treated by control isotype or anti-IL-17 (right). (G) Sirius red staining (left) and tissue collagen quantification (right) in the liver from mice treated by control isotype or anti-IL-17 antibodies. (H) Representative microphotographs of Hematoxylin-Eosin staining showing non-tumor (NT) and tumor (T) areas, and quantification in mice treated with control isotype or anti-IL-17 antibodies (right). (I) Representative immunohistochemistry staining for AFP and signal quantification in peritumoral or intratumoral areas. (J) Representative CD133 immunostaining and signal quantification in peritumoral and intratumoral areas. (K) Absolute quantification of miR-122 by $q P C R$. Data represent mean $\pm S E M$ and $* P<0.05 * * P<0.01$ mice treated with anti-IL17 $(n=7)$ versus isotype control $(n=6)$ antibody.

Although the role of various cytokines in cancer initiation has been largely described, their ability to transform normal cells has not been clearly demonstrated yet. In fact, cytokines exert their potential mutagenic effect by favoring the production of highly reactive molecules containing oxygen/nitrogen that can directly damage DNA in hepatocytes [14]. Considering TNF- $\alpha$ and IL-6 as the main cytokines secreted by Kupffer cells (the resident macrophages in the liver) that control both hepatocyte proliferation and LPC expansion, the effect of these proinflammatory factors on the malignant transformation of LPCs into CSCs has been evaluated. Long-term treatment with TNF- $\alpha$, but not IL-6, induced transformation of WB-F344 rat LPC which exhibited potent tumorigenicity in NOD/SCID mice [13]. Interestingly, this chronic TNF-a exposure triggered aberrant expression of Ubiquitin D and Checkpoint Kinase 2, key mediators of mitosis that cause significant chromosomal instability. Similarly, in our study, we demonstrated both in vitro and in vivo that long-term IL-17 treatment promotes LPC transformation into CSCs with enhanced self-renewal capacity. Moreover, histological analysis of tumors originating from LPC and growing in IL-17 rich microenvironment revealed an acquired aggressive phenotype (Figure 6B). These results are consistent with recent studies showing similar effect of IL-17 on self-renewal capacity of CSCs in different type of cancers [39, 40, 42].

Chronic nonresolving inflammation and tissue damage participate not only to the activation of LPC facultative compartment, but also to their transformation into CSCs [5, 9, 13]. Sustained IL-17dependent inflammation favors pro-inflammatory response in almost all tissues [15], especially in injured liver tissue, in which IL-17 drives hepatic fibrosis and promotes LPC expansion [17] and may also contribute to tumor initiation, progression and metastasis [16]. Anti-inflammatory treatment would decrease the onset of HCC. Interestingly, patient having aspirin as a chronic treatment have shown lower risk of developing HCC [43].

Tumor-associated inflammation can lead to epigenetic alterations [26] and the dysregulated expression of miRNAs are functionally linked to CSC development and several types of cancer including liver cancer [44]. In this study, miRNome analysis identified dysregulated miRNAs which may be involved in IL-17-triggered transformation of LPCs into CSCs. We observed a sharp decrease of miR-122-5p expression in LPCs after long-term treatment with IL-17 in vitro. Interestingly miR-122, which represents approximately $70 \%$ of total hepatic miRNAs, plays a pivotal role in cholesterol metabolism and iron homeostasis. Its expression is frequently reduced or abolished in HCC, while miR-122 is considered as a tumor suppressor in the liver [27]. In addition, we have shown that miR-122 mimic transfection into LPCs was sufficient to abolish self-renewal capacities acquired after long-term-IL-17 pretreatment. This result is in accordance with a study showing that miR-122 expression is decreased in CSC/CD133 ${ }^{+}$cells from several hepatic cancer cell lines, and that miR-122 re-expression is associated with reduced self-renewal capacities [45]. Together our findings and those from the literature suggest that IL-17 can increase LPC self-renewal capacities by negatively regulating miR-122 expression. This could support the idea that miR-122 is an interesting target for cancer therapy and/or prevention by limiting CSC occurrence and their expansion [46, 47]. However, taking into account that miR-122 promotes $\mathrm{HCV}$ replication [48], restoring miR-122 expression could not constitute a promising therapeutic strategy since miR-122 mimics could enhance hepatitis C liver damage progression.

The underlying mechanisms of cytokinemediated epigenetic alterations that drive the conversion of LPCs into CSCs are not fully understood. Our previous study clearly revealed that BMOL cell treatment with IL-17 increased their proliferation and reduced mRNA and protein expression of $\mathrm{HNF} 4 \alpha$, the master regulator of hepatic differentiation. We also demonstrated that IL-17 treatment abolished hepatocyte nuclear factor 4 alpha $(\mathrm{HNF} 4 \alpha)$ expression in freshly isolated LPC from choline-deficient and ethionine-supplemented diet fed mice. There are three HNF $4 \alpha$ binding sites present in miR-122 promoter, which may explain how IL-17 decreases miR-122 expression through the suppression of the liver-specific $H N F 4 \alpha[49,50]$. Interestingly, we confirmed that long term exposure of human LPC HepaRG to IL-17 also decreased 
HNF4 $\alpha$ expression (Supplemental Figure 1). IL-17mediated reduction of miR-122 levels could also result from signal transducer and activator of transcription 3 (STAT3) activation. Indeed, IL-17 induces IL-6 production that in turn activates STAT3-dependant pathways, thereby promoting the proliferation of HepG2 cells in vitro and tumor growth after orthotopic liver transplantation [51]. Interestingly, the activation of a complex STAT3-HNF4 inflammatory loop has been evidenced in persistent HCV-infected HCC cell line that was associated with decreased transcription of miR-122 [52]. Together, these results support the fact that persistent IL-17-mediated STAT3 activation could induce a decrease of HNF $4 \alpha$

\section{Liver chronic inflammation}

IL-17 immune response

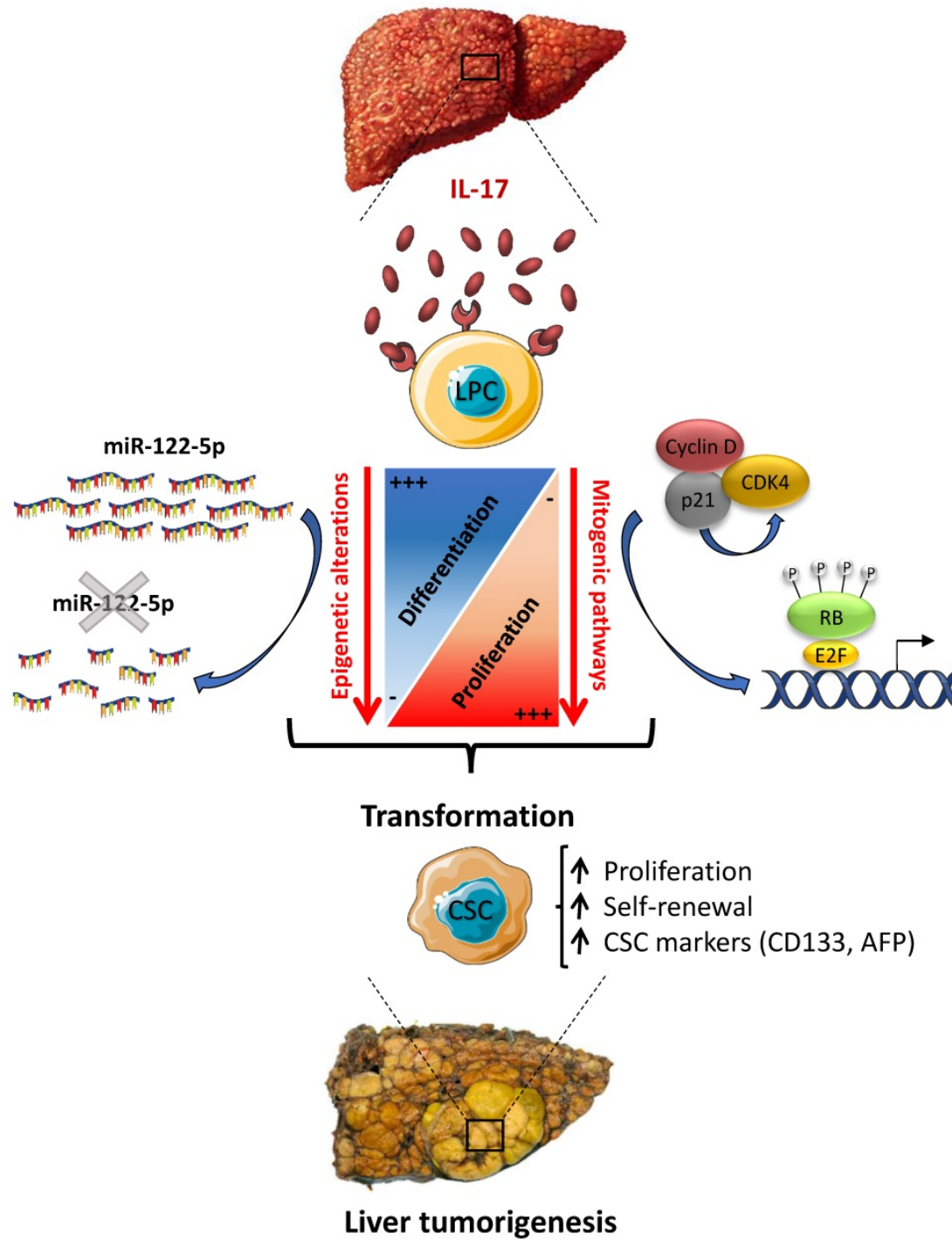

Figure 8. IL-17 triggers liver cancer development by favoring LPC malignant transformation into CSCs. IL-17 induces i) LPC de-differentiation by downregulating miR-122 expression and ii) enhances their proliferative and self-renewal capacities by increasing the expression of cell-cycle regulating factors. Altogether, these two pathways lead to LPC transformation into CSCs allowing them to trigger liver cancer development in vivo. expression and subsequent silencing of liver-specific miR-122.

In summary, the present work brings novel insights into the mechanisms of liver carcinogenesis, by showing an increased number of infiltrating IL-17-producing cells in preneoplastic cirrhotic livers that correlates with a transformation of LPCs into CSCs. Thus, a sustained IL-17 production might precede or accompany tumor development during chronic liver diseases. Our in vitro studies clearly showed that long-term stimulation of LPCs with IL-17 induces their transformation into liver CSCs. In this study, we evidenced that IL-17 promotes LPC stem-cell features and conversely attenuates their hepatocytic differentiation, by promoting mitogenic pathways and de-differentiation through miR-122 downregulation (Figure 8). As a conclusion, our study sheds light on how IL-17 cytokine could initiate primary liver cancer by transforming normal LPCs into tumor initiating cells through epigenetic reprogramming. Taken together, the data suggest that strategies aiming at neutralizing IL-17 production and/ or activity, that maintains miR-122 expression in the liver, should be assessed in clinical trials in patients with chronic liver diseases in order to prevent liver cancer initiation from LPC origin, including HCC, ICC and ICC-HCC mixed tumors.

\section{Abbreviations}

BMOL: Bipotential murine oval liver cell; $\mathrm{CCl}_{4}$ : Carbon tetrachloride; CK19: Cytokeratin 19; CSC: Cancer Stem cell; DEN: Diethylnitrosamine; DR: Ductular reaction; HCC: Hepatocellular carcinoma; ICC: Intrahepatic cholangiocarcinoma; IL-17: Interleukin-17A; LPC: Liver progenitor cell; SR: Sirius red.

\section{Supplementary Material}

Supplementary figures. https://www.ijbs.com/v18p1944s1. pdf

\section{Acknowledgements}

\section{Financial support statement}

This work was supported by the INSERM, the Université Paris-Est 
Créteil, the Institut Universitaire de France (IUF). IG and $\mathrm{CM}$ were recipient of a fellowship from the French Ministry of Higher Education, Research and Innovation.

\section{Author Contributions}

IG, CM, AR and AB performed experimental work and the animal procedures and prepared the manuscript. TCN and JC performed histopathological analysis. CR and VD helped with NGS and bioinformatics analyses. BR, AG, YAA edited the manuscript. AL, JMP, JC and AB helped analyze the data and edited the manuscript. FL designed and supervised the study and wrote the manuscript.

\section{Competing Interests}

The authors have declared that no competing interest exists.

\section{References}

1. Sung H, Ferlay J, Siegel RL, Laversanne M, Soerjomataram I, Jemal A, et al. Global Cancer Statistics 2020: GLOBOCAN Estimates of Incidence and Mortality Worldwide for 36 Cancers in 185 Countries. CA Cancer J Clin. 2021; 71: 209-49.

2. Marquardt JU, Andersen JB, Thorgeirsson SS. Functional and genetic deconstruction of the cellular origin in liver cancer. Nat Rev Cancer. 2015; 15: 653-67.

3. Ferlay J, Colombet M, Soerjomataram I, Mathers C, Parkin DM, Pineros M, et al. Estimating the global cancer incidence and mortality in 2018: GLOBOCAN sources and methods. Int J Cancer. 2019; 144: 1941-53.

4. Thorgeirsson SS. Stemness and reprogramming in liver cancer. Hepatology. 2016; 63: 1068-70.

5. Sia D, Villanueva A, Friedman SL, Llovet JM. Liver Cancer Cell of Origin, Molecular Class, and Effects on Patient Prognosis. Gastroenterology. 2017; 152: 745-61.

6. Batlle E, Clevers H. Cancer stem cells revisited. Nat Med. 2017; 23: 1124-34

7. Nio K, Yamashita T, Kaneko S. The evolving concept of liver cancer stem cells. Mol Cancer. 2017; 16: 4.

8. Wu Y, Zhang J, Zhang X, Zhou H, Liu G, Li Q. Cancer Stem Cells: A Potential Breakthrough in HCC-Targeted Therapy. Front Pharmacol. 2020; 11: 198.

9. Lukacs-Kornek V, Lammert F. The progenitor cell dilemma: Cellular and functional heterogeneity in assistance or escalation of liver injury. J Hepatol. 2017; 66: 619-30

10. Hou XJ, Ye F, Li XY, Liu WT, Jing YY, Han ZP, et al. Immune response involved in liver damage and the activation of hepatic progenitor cells during liver tumorigenesis. Cell Immunol. 2018; 326: 52-9.

11. Durnez A, Verslype C, Nevens F, Fevery J, Aerts R, Pirenne J, et al. The clinicopathological and prognostic relevance of cytokeratin 7 and 19 expression in hepatocellular carcinoma. A possible progenitor cell origin. Histopathology. 2006; 49: 138-51.

12. Komuta M, Spee B, Vander Borght S, De Vos R, Verslype C, Aerts R, et al. Clinicopathological study on cholangiolocellular carcinoma suggesting hepatic progenitor cell origin. Hepatology. 2008; 47: 1544-56.

13. Li XF, Chen $\mathrm{C}$, Xiang DM, Qu L, Sun $\mathrm{W}, \mathrm{Lu} \mathrm{XY}$, et al. Chronic inflammation-elicited liver progenitor cell conversion to liver cancer stem cell with clinical significance. Hepatology. 2017; 66: 1934-51.

14. Leone V, Ali A, Weber A, Tschaharganeh DF, Heikenwalder M. Liver Inflammation and Hepatobiliary Cancers. Trends Cancer. 2021.

15. Drescher HK, Bartsch LM, Weiskirchen S, Weiskirchen R. Intrahepatic TH17/TReg Cells in Homeostasis and Disease-It's All About the Balance. Front Pharmacol. 2020; 11: 588436.

16. Zhao J, Chen $\mathrm{X}$, Herjan $\mathrm{T}, \mathrm{Li} X$. The role of interleukin-17 in tumor development and progression. J Exp Med. 2020; 217.

17. Guillot A, Gasmi I, Brouillet A, Ait-Ahmed Y, Calderaro J, Ruiz I, et al. Interleukins-17 and 27 promote liver regeneration by sequentially inducing progenitor cell expansion and differentiation. Hepatol Commun. 2018; 2: 329-43.

18. Liao R, Sun J, Wu H, Yi Y, Wang JX, He HW, et al. High expression of IL-17 and IL-17RE associate with poor prognosis of hepatocellular carcinoma. J Exp Clin Cancer Res. 2013; 32: 3.

19. Sun C, Kono H, Furuya S, Hara M, Hirayama K, Akazawa Y, et al. Interleukin-17A Plays a Pivotal Role in Chemically Induced Hepatocellular Carcinoma in Mice. Dig Dis Sci. 2016; 61: 474-88.
20. Liang KH, Lai MW, Lin YH, Chu YD, Lin CL, Lin WR, et al. Plasma interleukin-17 and alpha-fetoprotein combination effectively predicts imminent hepatocellular carcinoma occurrence in liver cirrhotic patients. BMC Gastroenterol. 2021; 21: 177.

21. Bankhead P, Loughrey MB, Fernandez JA, Dombrowski Y, McArt DG, Dunne $\mathrm{PD}$, et al. QuPath: Open source software for digital pathology image analysis. Scientific reports. 2017; 7: 16878 .

22. W. FTAaR. The ImageJ user guide. June 2012 ed: Tech. Rep. IJ 1.46,; 2012. p. "The ImageJ user guide,"

23. Gao J, Aksoy BA, Dogrusoz U, Dresdner G, Gross B, Sumer SO, et al. Integrative analysis of complex cancer genomics and clinical profiles using the cBioPortal. Sci Signal. 2013; 6: pl1.

24. Hoadley KA, Yau C, Hinoue T, Wolf DM, Lazar AJ, Drill E, et al. Cell-ofOrigin Patterns Dominate the Molecular Classification of 10,000 Tumors from 33 Types of Cancer. Cell. 2018; 173: 291-304 e6.

25. Warfel NA, El-Deiry WS. p21WAF1 and tumourigenesis: 20 years after. Curr Opin Oncol. 2013; 25: 52-8.

26. Poli V, Fagnocchi L, Zippo A. Tumorigenic Cell Reprogramming and Cancer Plasticity: Interplay between Signaling, Microenvironment, and Epigenetics. Stem Cells Int. 2018; 2018: 4598195.

27. Zeisel MB, Pfeffer S, Baumert TF. miR-122 acts as a tumor suppressor in hepatocarcinogenesis in vivo. J Hepatol. 2013; 58: 821-3.

28. Xu F, Yi J, Wang F, Wang W, Wang Z, Xue J, et al. Involvement of soluble B7-H3 in combination with the serum inflammatory cytokines interleukin-17, -8 and -6 in the diagnosis of hepatocellular carcinoma. Oncol Lett. 2017; 14: 8138-43.

29. Lee K, Lee KB, Jung HY, Yi NJ, Lee KW, Suh KS, et al. The correlation between poor prognosis and increased yes-associated protein 1 expression in keratin 19 expressing hepatocellular carcinomas and cholangiocarcinomas. BMC Cancer. 2017; 17: 441.

30. Song W, Li H, Tao K, Li R, Song Z, Zhao Q, et al. Expression and clinical significance of the stem cell marker CD133 in hepatocellular carcinoma. Int J Clin Pract. 2008; 62: 1212-8

31. Cai X, Li J, Yuan X, Xiao J, Dooley S, Wan X, et al. CD133 expression in cancer cells predicts poor prognosis of non-mucin producing intrahepatic cholangiocarcinoma. J Transl Med. 2018; 16: 50

32. Khosla R, Rastogi A, Ramakrishna G, Pamecha V, Mukhopadhyay A, Vasudevan M, et al. EpCAM+ Liver Cancer Stem-Like Cells Exhibiting Autocrine Wnt Signaling Potentially Originate in Cirrhotic Patients. Stem Cells Transl Med. 2017; 6: 807-18.

33. Krause J, von Felden J, Casar C, Frundt TW, Galaski J, Schmidt C, et al Hepatocellular carcinoma: Intratumoral EpCAM-positive cancer stem cell heterogeneity identifies high-risk tumor subtype. BMC Cancer. 2020; 20: 1130.

34. Zeng Y, Zhang Q, Wang $\mathrm{H}$, Lu M, Kong $\mathrm{H}$, Zhang $\mathrm{Y}$, et al. Prognostic significance of interleukin-17 in solid tumors: a meta-analysis. Int J Clin Exp Med. 2015; 8: 10515-36

35. Ma HY, Yamamoto G, Xu J, Liu X, Karin D, Kim JY, et al. IL-17 signaling in steatotic hepatocytes and macrophages promotes hepatocellular carcinoma in alcohol-related liver disease. J Hepatol. 2020; 72: 946-59.

36. Housseau F, Wu S, Wick EC, Fan H, Wu X, Llosa NJ, et al. Redundant Innate and Adaptive Sources of IL17 Production Drive Colon Tumorigenesis. Cancer Res. 2016; 76: 2115-24.

37. Jiang YX, Yang SW, Li PA, Luo X, Li ZY, Hao YX, et al. The promotion of the transformation of quiescent gastric cancer stem cells by IL-17 and the underlying mechanisms. Oncogene. 2017; 36: 1256-64

38. Wu L, Chen X, Zhao J, Martin B, Zepp JA, Ko JS, et al. A novel IL-17 signaling pathway controlling keratinocyte proliferation and tumorigenesis via the TRAF4-ERK5 axis. J Exp Med. 2015; 212: 1571-87.

39. Xiang $\mathrm{T}$, Long $\mathrm{H}, \mathrm{He} \mathrm{L}, \mathrm{Han} \mathrm{X}$, Lin $\mathrm{K}$, Liang $\mathrm{Z}$, et al. Interleukin-17 produced by tumor microenvironment promotes self-renewal of CD133+ cancer stem-like cells in ovarian cancer. Oncogene. 2015; 34: 165-76.

40. Zhang Y, Zoltan M, Riquelme E, Xu H, Sahin I, Castro-Pando S, et al. Immune Cell Production of Interleukin 17 Induces Stem Cell Features of Pancreatic Intraepithelial Neoplasia Cells. Gastroenterology. 2018.

41. Mombelli S, Cochaud S, Merrouche Y, Garbar C, Antonicelli F, Laprevotte E, et al. IL-17A and its homologs IL-25/IL-17E recruit the c-RAF/S6 kinase pathway and the generation of pro-oncogenic LMW-E in breast cancer cells. Scientific reports. 2015; 5: 11874 .

42. Zhang Q, Liu S, Parajuli KR, Zhang W, Zhang K, Mo Z, et al. Interleukin-17 promotes prostate cancer via MMP7-induced epithelial-to-mesenchymal transition. Oncogene. 2017; 36: 687-99.

43. Simon TG, Duberg AS, Aleman S, Chung RT, Chan AT, Ludvigsson JF. Association of Aspirin with Hepatocellular Carcinoma and Liver-Related Mortality. N Engl J Med. 2020; 382: 1018-28.

44. Hwang GR, Yuen JG, Ju J. Roles of microRNAs in Gastrointestinal Cancer Stem Cell Resistance and Therapeutic Development. Int J Mol Sci. 2021; 22.

45. Song K, Kwon H, Han C, Zhang J, Dash S, Lim K, et al. Active glycolytic metabolism in CD133(+) hepatocellular cancer stem cells: regulation by MIR-122. Oncotarget. 2015; 6: 40822-35.

46. Boix L, Lopez-Oliva JM, Rhodes AC, Bruix J. Restoring mir122 in human stem-like hepatocarcinoma cells, prompts tumor dormancy through smad-independent TGF-beta pathway. Oncotarget. 2016.

47. Dhungel B, Ramlogan-Steel CA, Layton CJ, Steel JC. miRNA122a regulation of gene therapy vectors targeting hepatocellular cancer stem cells. Oncotarget. 2018; 9: 23577-88. 
48. Jopling CL, Yi M, Lancaster AM, Lemon SM, Sarnow P. Modulation of hepatitis C virus RNA abundance by a liver-specific MicroRNA. Science. 2005; 309: 1577-81.

49. Li ZY, Xi Y, Zhu WN, Zeng C, Zhang ZQ, Guo ZC, et al. Positive regulation of hepatic miR-122 expression by HNF4a. J Hepatol. 2011; 55: 602-11.

50. Lu H, Lei X, Liu J, Klaassen C. Regulation of hepatic microRNA expression by hepatocyte nuclear factor 4 alpha. World J Hepatol. 2017; 9: 191-208.

51. Hu Z, Luo D, Wang D, Ma L, Zhao Y, Li L. IL-17 Activates the IL-6/STAT3 Signal Pathway in the Proliferation of Hepatitis B Virus-Related Hepatocellular Carcinoma. Cell Physiol Biochem. 2017; 43: 2379-90.

52. Aydin Y, Kurt R, Song K, Lin D, Osman H, Youngquist B, et al. Hepatic Stress Response in HCV Infection Promotes STAT3-Mediated Inhibition of HNF4A-miR-122 Feedback Loop in Liver Fibrosis and Cancer Progression. Cancers (Basel). 2019; 11.

\section{Author Biography}

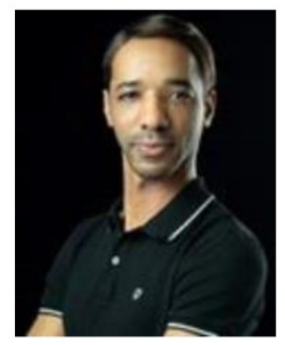

Dr. Fouad Lafdil is Full Professor at the University of Paris-Est and is leading research projects on liver pathophysiology at Henri Mondor Hospital since 2009. Prof. LAFDIL acquired a solid background in liver immunology field as postdoctoral fellow at the National Institutes of Health (NIH/NIAAA). He is currently developing novel research projects aiming to understanding the role of the immune microenvironment in primary liver cancers including hepatocellular carcinoma (HCC) initiation, progression, and resistance to current anticancer therapies. He is combining clinical analysis on patients with chronic liver diseases and in vivo approaches on several murine models of liver diseases. One of his goals is to identify novel anticancer strategies based on immunotherapy. He is a member of the French Association for the Study of the Liver (AFEF) since 2012. He also awarded a high distinction of scientific excellence as a member of the Institute of French Universities (IUF) since 2016. 